\title{
Cardiac Spiral Wave Drifting Due to Spatial Temperature Gradients - a Numerical Study
}

Guy Malki, and Sharon Zlochiver

Department of Biomedical Engineering Department, Faculty of Engineering, Tel-Aviv University, Ramat-Aviv, Tel-Aviv, 69978, Israel

\section{Correspondence:}

Sharon Zlochiver, Ph.D.

Department of Biomedical Engineering,

Faculty of Engineering,

Tel-Aviv University, Ramat-Aviv, Tel-Aviv, 69978, Israel

Tel: +972-3-6409940 Fax: +972-3-6407939

E-mail: sharonz@eng.tau.ac.il 


\section{ABSTRACT}

Cardiac rotors are believed to be a major driver source of persistent atrial fibrillation $(A F)$, and their spatiotemporal characterization is essential for successful ablation procedures. However, electrograms guided ablation have not been proven to have benefit over empirical ablation thus far, and there is a strong need of improving the localization of cardiac arrhythmogenic targets for ablation. A new approach for characterize rotors is proposed that is based on induced spatial temperature gradients (STGs), and investigated by theoretical study using numerical simulations. We hypothesize that such gradients will cause rotor drifting due to induced spatial heterogeneity in excitability, so that rotors could be driven towards the ablating probe. Numerical simulations were conducted in single cell and 2D atrial models using AF remodeled kinetics. STGs were applied either linearly on the entire tissue or as a small local perturbation, and the major ion channel rate constants were adjusted following Arrhenius equation. In the AF-remodeled single cell, recovery time increased exponentially with decreasing temperatures, despite the marginal effect of temperature on the action potential duration. In 2D models, spiral waves drifted with drifting velocity components affected by both temperature gradient direction and the spiral wave rotation direction. Overall, spiral waves drifted towards the colder tissue region associated with global minimum of excitability. A local perturbation with a temperature of $\mathrm{T}=28^{\circ} \mathrm{C}$ was found optimal for spiral wave attraction for the studied conditions. This work provides a preliminary proof-of-concept for a potential prospective technique for rotor attraction. We envision that the insights from this study will be utilize in the future in the design of a new methodology for AF characterization and termination during ablation procedures. meandering and drifting 


\section{INTRODUCTION}

31 Atrial fibrillation (AF) is the most common sustained cardiac arrhythmia, 32 affecting more than $10 \%$ of the elderly population [1]. AF is characterized by 33 rapid and irregular activation of the atrium, and is often the result of fibrillatory 34 conduction maintained by the existence of one or few organized "mother rotors", or alternatively by the existence of focal ectopic sources, [2-3]. Pharmacological treatment for AF includes antiarrhythmic drugs that are either rate or rhythm control. These effects are achieved by various ionic mechanisms altering the electrophysiological properties of the membrane voltage at either depolarization or repolarization phases, e.g. by reducing cellular excitability, or by increasing the refractory period. Nonetheless, as pharmacological treatment for AF shows limited success [4] and due to its possible long term negative side-effects, surgical ablation procedures have become attractive for curing AF in symptomatic patients. These procedures try to isolate and annihilate arrhythmogenic sources in the atrial tissue by inducing permanent tissue ablation, typically by delivering radiofrequency (RF) energy. While unguided, empirical ablation is practiced in some procedures, it often results in redundant applications of RF energy that may cause inadvertent injury and thromboembolic complications in the tissue. Thus, the correct characterization of the arrhythmogenic sources (rotors vs. focal activity) and their localization are of the utmost importance for a successful outcome of the ablation procedure, and several electrogramguided ablation techniques have been recently proposed or utilized for that purpose [5-6]. Those techniques are based on either time-domain or frequency-domain analysis, and constructed by single electrogram analysis, or multiple, normally simultaneously-recorded electrograms, to provide spatiotemporal characterization of the electrical activity in the atrial tissue. Electrogram-derived indices, such as complex fractionated atrial electrograms (CFAEs) [7-8] and dominant frequency (DF) [9-10], have been proposed as guide to perform electrogram-based ablation, and recently logical combination

60 of rate and regularity measures has been developed to achieve computational 61 mapping of the atrial sources [6]. Phase analysis using phase singularity evaluation has been used to characterize rotors and their pivot points $[5,11]$. 
63 Though, none of these practices were successfully implemented in clinical 64 settings because of various limitations in their ability to accurately characterize the arrhythmogenic source zones due to noise, misleading phase and activation times that distort the reconstructed maps [11]. Moreover, novel techniques that involve more advanced signal processing methodologies to locate the pivot points of persistent rotors were proposed lately, including principal component analysis [12], and spatial Shannon entropy measurement [13]. Nevertheless, due to the yet poor understanding and the ambiguity of the correlations between the underlying arrhythmogenic activity and its electrogram manifestation, reported ablation success rates are similar for electrogram-guided as for empirical ablation procedures [4, 14]. Therefore, there is a clear need for new approaches to improve guided ablation procedures by better detection and characterization of arrhythmogenic drivers in order to minimize the destruction of unnecessary healthy tissue and allow for higher long-term success rates of such procedures. Here we propose a novel strategy for addressing this need for the specific cardiac rotors arrhythmogenic drivers in persistent AF patients, which is based on artificial induction of temperature gradients in the atrial tissue. The effect of temperature on the biophysical properties of various biological tissues has been long studied. Temperature sustains direct impact on biological processes, thus influencing ion channel kinetics, action potential (AP) morphology and other electrophysiological properties via its effects on the rate constants of chemical reactions and sub-cellular biological processes [15-17]. These effects are commonly modelled by the multiplication of the relevant reaction rate constants (e.g., ion channel gating variables) by a scaling factor, which sustains a power-law relationship with temperature following Arrhenius' law [18]. Consequently, e.g., a temperature increase results in faster gating kinetics leading to a decrease in the cardiac AP duration (APD), while induced hypothermia on the other hand inhibits the 92 dynamics of the membrane currents, effectively prolonging the APD [19]. 93 Several studies have related temperature changes to cardiac pathologies 94 associated with impaired electrical conduction [20-23]. The contribution of 95 temperature on pro-arrhythmic APD restitution properties and alternans 96 formation was recently studied by Fenton et al. [24-25] using simplified 
97 ventricular model with thermoelectric coupling. Their studies showed that 98 alternans and conduction block onset occur in higher cycle lengths as temperature decreases, and that the minimum tissue size required to sustain hypothermic ventricular fibrillation is temperature dependent. Nevertheless, ionic mechanisms explaining the effect of temperature could not be pursued due to the abstract modelling approach. Finally, Yamazaki et al. [26] demonstrated in an experimental study using rabbit hearts that regional cooling within the left ventricle facilitates termination due to collisions with boundaries of spiral-wave re-entry through unpinning of rotors and drift toward the cooled region. Here too, these important findings lack detailed biophysical and theoretical elucidation, and are supported by experimental evidence only.

108 In this study we will examine a possible positive effect of externally applied spatial temperature gradients (STGs) on atrial tissues activated by a dominant rotor - induced controlled drifting. While the exact mechanism remains unclear, both experimental and numerical studies show rotor drifting and meandering due to various types of spatial gradients in both biophysical and anatomical properties [27-29]. Rotors have been shown to drift towards regions of lower excitability of various origins, including heterogeneity in sodium channel availability [30-31], or in IK1 channel density [32]. Based on these results, we postulate that externally applied STGs will enable controlled rotor drifting by a similar mechanism of spatial heterogeneity in atrial excitability. The aim of this study is to establish the theory underlying the relationship between temperature gradients, rotor drifting and tissue excitability. We envision that in the future our approach can indeed promote

121 relevant clinical applications during ablation procedures.

\section{METHODS}

\section{A. Biophysical Modeling}

124 Atrial electrical activity was simulated in either a single cell or a $30 \mathrm{~mm} \times 30$

$125 \mathrm{~mm} 2 \mathrm{D}$ tissue by solving the following reaction-diffusion equation by adopting

126 the mono-domain formalism and under the approximation of tissue isotropy:

$$
\partial V / \partial t=-\left(I_{\text {ion }}+I_{\text {stim }}\right) / C_{m}+\nabla \cdot(D \nabla V)
$$


128 where $V[\mathrm{mV}]$ is the transmembrane voltage, $C_{m}\left[\mu \mathrm{F} / \mathrm{cm}^{2}\right]$ is the membrane 129 capacitance per unit area, $I_{\text {stim }}$ and $I_{\text {ion }}\left[\mu \mathrm{A} / \mathrm{cm}^{2}\right]$ are the external stimulation 130 and membrane ionic currents, respectively, and $D\left[\mathrm{~mm}^{2} / \mathrm{ms}\right]$ is the diffusion 131 coefficient. Human atrial kinetics were employed for calculating $I_{\text {ion }}$ using the 132 Courtemanche-Ramirez-Nattel (CRN) model [33]. In most simulations, the 133 CRN model was modified to account for chronic AF remodeling by down134 regulating the channel densities of the transient outward $\mathrm{K}+$ current $\left(I_{t o}\right)$ by $13550 \%$, the ultra-rapid delayed rectifier $\mathrm{K}+$ current $\left(I_{K u r}\right)$ by $50 \%$, the L-type $136 \mathrm{Ca} 2+$ current $\left(I_{C a-L}\right)$ by $70 \%$, and by increasing the density of the inward 137 rectifier $\mathrm{K}+$ current $\left(I_{K 1}\right)$ by $100 \%$, according to previous publications [30, 34]. 138 The diffusion coefficient was adjusted to $D=0.03 \mathrm{~mm}^{2} / \mathrm{ms}$ to achieve planar 139 conduction velocity (CV) of $\sim 0.4 \mathrm{~m} / \mathrm{s}$. The effect of temperature on the model 140 kinetics was incorporated by multiplying the rate constants $\alpha\left(37^{\circ} \mathrm{C}\right)$ and $141 \beta\left(37^{\circ} \mathrm{C}\right)$ of the gating variables of several ion channels at a reference 142 temperature of $37^{\circ} \mathrm{C}$ by the temperature adjustment factor $Q(T)$ following 143 Arrhenius equation, as originally proposed by Hodgkin \& Katz [15]:

144

146

150

151

152

153

154

155

156

157

158

159

$$
\begin{aligned}
& \alpha(T)=\alpha\left(37^{\circ} \mathrm{C}\right) \cdot Q(T) \\
& \beta(T)=\beta\left(37^{\circ} \mathrm{C}\right) \cdot Q(T) \\
& Q(T)=Q_{10}^{\frac{T-37^{\circ} \mathrm{C}}{10^{\circ} \mathrm{C}}}
\end{aligned}
$$

where $T\left[{ }^{\circ} \mathrm{C}\right]$ is the local tissue temperature, and $Q_{10}$ is the temperature coefficient defining the ratio by which the rate constants increase following a temperature increase of $10^{\circ} \mathrm{C}$. Equations (2-4) were applied to the six main ion channel currents as detailed in Table 1. Equation (1) was solved numerically by employing forward Euler integration in time and the finite difference method in space, using temporal and spatial resolutions of $\Delta t=2.5$ $\mu$ s and $\Delta h=0.1 \mathrm{~mm}$, respectively. Those values were set after ensuring convergence of the simulation data by comparison to those obtained with finer resolution values.

\section{B. Simulation Configuration}

Single cell simulations were conducted to study the effect of temperature on basic electrophysiological properties. Spiral waves were established in a 
160 uniform $2 \mathrm{D}$ geometry with a fixed temperature of $37^{\circ} \mathrm{C}$, using the $\mathrm{S} 1-\mathrm{S} 2$ cross-

161 field stimulation. This protocol induced a counter clockwise or clockwise

162 rotating spiral wave in the middle of the tissue with a dominant activation

163 frequency of $8.1 \mathrm{~Hz}$. Five second long electrical activity was simulated prior to

164 any application of spatial temperature gradients (STGs) to ensure initial

165 stability of the spiral wave. At the end of that stabilization period, STGs of

166 either one of two types were applied (see Fig. 1): 1) linearly changing

167 temperature gradients along the $\mathrm{y}$-axis between $T_{1}=37^{\circ} \mathrm{C}$ and varying $T_{2}$

168 (between $30^{\circ} \mathrm{C}$ and $44^{\circ} \mathrm{C}$ ), so that $T(y=0)=37^{\circ} \mathrm{C}$ and $T(y=30 \mathrm{~mm})=T_{2}$.

169 This configuration allowed the investigation of basic spiral wave drifting

170 mechanisms under controlled temperature gradients; 2) local circular

171 temperature perturbation with a radius of $1 \mathrm{~mm}$, typical for AF ablation

172 catheters. This configuration was intended to mimic the potential clinical

173 application of an external heat source catheter during an EPS procedure. Two

174 types of perturbation configurations were simulated: in the first type, the

175 location of the perturbation center was kept fixed at $8 \mathrm{~mm}$ from the initial

176 location of the spiral wave core center, but its temperature was varied

177 between $20^{\circ} \mathrm{C}$ and $36^{\circ} \mathrm{C}$. In the second type, the perturbation temperature was

178 fixed at $28^{\circ} \mathrm{C}$, but its distance from the core of the spiral wave was changed

179 between $5 \mathrm{~mm}$ and $11 \mathrm{~mm}$. To account for the heat distribution due to the

180 application of a circular heat perturbation, the following bio-heat transfer

181 equation was numerically solved in steady-state [40]:

$182 \nabla \cdot\left(K_{t} \cdot \nabla T\right)+Q_{b i o}=0$,

183 where $K_{t}=0.7 \mathrm{~W} /(\mathrm{m} \cdot \mathrm{K})$ is the atrial tissue thermal conductivity, and $Q_{b i o}$

$184\left[\mathrm{~W} / \mathrm{m}^{3}\right]$ is a source term representing the blood perfusion, and is equal to:

$185 \quad Q_{b i o}=\rho_{b} C_{b} w_{b} \cdot\left(T_{b}-T\right)$,

186 where $\rho_{b}=1080 \mathrm{~kg} / \mathrm{m}^{3}$ is the blood density, $C_{b}=3600 \mathrm{~J} /(\mathrm{kg} \cdot \mathrm{K})$ is the specific

187 heat capacity of the blood, $w_{b}=1 \mathrm{~Hz}$ is the perfusion rate and $T_{b}=37^{\circ} \mathrm{C}$ is

188 the arterial blood temperature. Equations (5-6) were solved numerically for

189 each perturbation configuration in Fig. 1 using COMSOL finite element

190 software (COMSOL, Burlington, MA).

191 
192

193

194

195

196

197

198

199

200

201

202

203

204

205

206

207

208

209

210

211

212

213

214

215

216

217

218

219

220

221

222

223

\section{Technical Aspects and Data Analysis}

All simulations were performed using $\mathrm{C}++$ code on a high-performance cluster computer (Altix X86-PTO; Silicon Graphics International, Milpitas, CA) with a master node (eight cores, Xeon $2.5 \mathrm{GHz}$ processor; Intel, Santa Clara, CA) and up to five computational nodes (60cores, Xeon $2.8 \mathrm{GHz}$; Intel). Data analysis and visualization were performed with MATLAB R2014b (The MathWorks, Natick, MA). In order to investigate the spiral wave stability and its rate of drifting, tip trajectory was automatically tracked following Nayak et al. [41]. Briefly, this method locates the singularity point as the intersection region between a certain iso-potential range (in our case between $-40 \mathrm{mV}$ and $-15 \mathrm{mV}$, range $\mathrm{A}$ ) and the range $\partial V / \partial t=0$ (range $\mathrm{B}$ ). To avoid incorrectly detecting overlapping points, we first employed a $3 \times 3$ pixel median filter over the range $A$ to fill in small gaps, and then defined the singularity point map as the overlapping areas of the filtered range $A$ and range $B$ (range $C$ ). Since in most cases more than one potential singular point was detected in range $\mathrm{C}$, we used the exponentially-weighted moving average (EWMA) method to choose the most likely singular point in that specific time frame. In the EWMA method, we first set exponentially decreasing weights to all singular points detected in previous time frames, with the highest weight given to the most recent detected singular point. We then calculated a projected location of the next singularity point by employing a moving average on the exponentially weighted past singularity point locations. Lastly, we selected the singularity point out of range $\mathrm{C}$ that was the closest to the projected location. Spatial excitability distribution was represented by the sodium channel availability, given by the product of its fast $(h)$ and slow $(j)$ inactivation gates [2].

\section{RESULTS}

\section{Single Cell Simulations and Model Validation}

The effects of temperature on the AP morphology and on cellular excitability are shown in Fig. 2, for both standard CRN model parameters (panel A) and for chronic AF modifications (panel B). Temperature variations sustained significant effects in standard model parameters, as can be seen from a detailed characterization of the temperature dependence in the ionic model in 
224 panel $\mathrm{C}$, which presenting the analysis of these parameters at a vast range of 225 temperatures that does not produce irreversible tissue damage $\left(20^{\circ} \mathrm{C}-42^{\circ} \mathrm{C}\right)$.

226 Both APD at 90\% repolarization (APD90), as well as recovery time from 227 inactivation, calculated by the time interval between sodium channel 228 availability $(h \times j)$ levels of 0.1 and 0.9 , (panel C, upper and lower left columns, 229 respectively), decreased with increasing temperatures. These results are a 230 direct consequence of the increasing rate constants with increased 231 temperatures (2-3), and support our hypothesis assumption that low 232 temperature regions in the tissue exhibit reduced excitability. When chronic 233 AF modifications were implemented, the effect of temperature variations on 234 the AP morphology and duration was minor. Notwithstanding, temperature 235 effect on the recovery time from inactivation was still substantial, signifying 236 that the correlation between low temperature and reduced excitability holds in 237 chronic AF conditions as well. The AP amplitude as function of temperature 238 (panel C, upper right plot) exhibits a similar behavior, in which decreased as 239 the temperature increasing. This observation attests that the sodium channel 240 rate constant effected the sodium gating to open more slowly in a colder 241 temperature, and therefore amplified the depolarization process. On the other 242 hand, CV (panel C, bottom right plot) raised as the temperature increased, 243 because of the growing rate constants, which yielded to rapider propagation.

244 Furthermore, spiral wave dynamics with constant tissue temperature have 245 been explored, as can be seen in Fig. S1. Three spiral wave properties were 246 analyzed to describe the tip meandering as function of temperature: the fast 247 frequency that determines the interval between consecutive local excitation, 248 also called the activation frequency $\left(f_{1}\right)$; the slow frequency that determines 249 the rotation rate of the spiral wave tip, denoted as the meander frequency $250\left(f_{2}\right)$; and the mean tip velocity during single and complete rotation cycle. All of 251 them are aligned with the model predications, so that the spiral wave 252 dynamics are reduced, became slower and have lower frequencies, as the 253 tissue temperature is decreasing. 
255 Tissue Simulations - Linear Temperature Gradients

$2562 \mathrm{D}$ simulations were conducted for a $30 \mathrm{~mm} \times 30 \mathrm{~mm}$ atrial tissue model 257 using the CRN model with chronic AF modifications. Vertical linear STGs 258 were applied as detailed in the methods section. Fig. 3A shows typical tip 259 trajectories due to either a positive STG $\left(T_{2}=40^{\circ} \mathrm{C}>37^{\circ} \mathrm{C}\right.$, in green) or a 260 negative STG $\left(T_{2}=34^{\circ} \mathrm{C}<37^{\circ} \mathrm{C}\right.$, in blue), for both clockwise and counter261 clockwise rotation (light and dark traces, respectively). It can be seen that the 262 vertical drifting component was directed opposite to the gradient direction $263(d T / d y)$, while the horizontal component was directed according to the sign of 264 the product $(d T / d y) \cdot R$, where $R$ obtains the values of +1 or -1 for clockwise 265 and counter-clockwise rotations, respectively. For example, a clockwise 266 rotating spiral wave $(R=1)$ with $T_{2}=34^{\circ} \mathrm{C}(d T / d y<0)$ drifted towards the 267 upper-left corner (light blue trace) of the tissue due to a positive vertical 268 component $(-d T / d y>0)$ and a negative horizontal component $(d T / d y \cdot R<$ 2690 ). Fig. 3B illustrates the spatiotemporal tip trajectory for a clockwise rotating 270 spiral wave and for linear STGs different by their $T_{2}$ varying between $30^{\circ} \mathrm{C}$ and $27144^{\circ} \mathrm{C}$. Mean tip trajectories (bold lines) were calculated by applying spatial 272 trajectory averaging using sliding temporal windows of durations that 273 corresponded with the time required by the tip to complete a single rosette274 like cycle. The mean vertical and horizontal drifting velocities were calculated 275 from the mean tip trajectories by extracting the total $y$-axis and $x$-axis tip 276 translation during the first 3 seconds of activity, and are shown as a function 277 of $(d T / d y)$ in Fig. 3C. Fig. 3D represents the angle between the drift velocity 278 vector and the gradient vector as a function of $(d T / d y)$. This analysis reveals 279 the effect of temperature on the drifting angle, and discovers a discontinuity at 280 zero gradient that was emphasized by studying smaller gradients in both 281 directions. It should be noted that at larger gradients (e.g. T2 $=30^{\circ} \mathrm{C}$ and $282 \mathrm{~T} 2=44^{\circ} \mathrm{C}$ ), in addition to the change in the drifting angle, the drifting trajectory 283 pattern is slightly altered after the initial drift due to interaction with the 284 boundaries. Thus, Figs. 3B-D demonstrate that wave drifting translation 285 monotonically and symmetrically depended on the difference $T_{2}-37^{\circ} \mathrm{C}$.

286 Next, we demonstrate that, in agreement with our hypothesis, spiral wave 287 drifting is due to the induction of spatial heterogeneity in tissue excitability 
288 following the application of the STG, such that drifting occurs from high to low 289 excitability regions. We focus on the case in which an STG with $T_{2}=30^{\circ} \mathrm{C}$ is 290 applied on a tissue with a clockwise rotating rotor at $\mathrm{t}=0$. As was shown in Fig. 291 3, the application of such STG resulted in a spiral wave diagonally drifting 292 towards the top-left corner of the tissue. The mean drifting trajectory is shown 293 in Fig. 4A, while the temporal evolution of the sodium channel availability 294 (represented by $h \times J$ ) is shown as a time-space plot in Fig. 4B along the 295 diagonal profile that matches the drifting trajectory (dashed line in Fig. 4A). In 296 this plot, the $x$-axis represents a normalized linear coordinate along the 297 diagonal profile (i.e., $p=0$ and $p=1$ represent the top-left and bottom-right 298 edges, respectively), the $y$-axis represents time, and the $h \times j$ values are coded 299 by color. The locations of the spiral wave pivot along the diagonal profile can 300 be easily visualized on the time-space plot as the thick, purple-colored line 301 (marked with an arrow), thus representing low sodium channel availability, 302 that starts close to the center $(p=0.5)$ at $t=0$, and ends at the top-left region of 303 the tissue $(p=0.16)$ where the drifting ends after $\sim 3 \mathrm{sec}$. Figure $4 \mathrm{C}$ presents a 304 zoomed-in view of part of the time-space plot, and demonstrates the 305 consistent imbalance in the $h_{x j}$ values between the two sides of the pivot 306 location during the drifting process. For each rotation cycle of the spiral wave 307 the sodium channel availability (hence excitability) to the left of the pivot is 308 lower than to its right (see markers on the inset in Fig. 4C). Hence the pivot 309 movement to the left indicates drifting of the spiral wave towards the region 310 with reduced excitability, i.e., the colder region. To further demonstrate this 311 idea, the 5 -sec long time average of the $h_{x j}$ values along the diagonal profile 312 is shown in Fig. 4D, with the beginning and ending pivot points marked by 313 dots. This figure clearly shows the pivot drifting towards the low excitability 314 region, or more precisely, to the location with globally minimum mean $h \times j$. To 315 understand the dynamical mechanism by which drifting was occurring towards 316 the low excitability (colder) region of the tissue, an activation map 317 corresponding to one spiral wave rotation during drifting is shown in Fig. 5. 318 This map shows wavefront activation isochronal lines with temporal intervals 319 of $10 \mathrm{~ms}$. The tip trajectory during that cycle is marked by the bold black line, 320 with the orange and green dots showing the tip initial and final locations, 321 respectively. The activation map clearly shows the net diagonal drifting 
322 towards the top-left corner during that cycle. During drifting from a hotter to a

323 colder region, the isochrones become denser and denser, indicating slowing

324 down of the wavefront due to propagation in a gradually less excitable region.

325 This also implies a longer drifting distance that was required for the wavefront

326 before being able to rotate back towards the hotter region, due to its reduced

327 capability to reenter in a low excitability region in comparison with its

328 increased capability to reenter in a high excitability region. The gradually

329 reducing tissue excitability during the spiral wave drifting towards the colder

330 region was a direct consequence of the longer recovery time associated with

331 the reduced local temperature (see Fig. 2C). Since the imposed STG was

332 linear, and as recovery time increases non-linearly but rather exponentially

333 with decreasing temperatures (Fig. 2C), this also contributed to a longer

334 required tip drifting distance when traveling towards a colder region in

335 comparison to travelling towards a hotter region before the local tissue was

336 sufficiently recovered to sustain a successful reentry. Hence, overall net

337 drifting was towards the colder region.

339 Tissue Simulations - Local Perturbations of Varying Temperatures

340 Next we studied the feasibility of a clockwise rotor attraction by a small

341 temperature perturbation, that may represent, e.g., the application of an

342 external cooling probe. Circular temperature perturbations with a radius of

$3431 \mathrm{~mm}$ were modeled as described in the methods section, with a constant

344 atrial background temperature of $T_{1}=37^{\circ} \mathrm{C}$. Following the results in the

345 previous section, only perturbations having temperature $T_{2}<T_{1}$ were modeled

346 since spiral waves drift from high to low temperature regions. Fig. 6A shows

347 the mean tip trajectories during 50 seconds of activity when applying

348 perturbations with various temperatures between $20^{\circ} \mathrm{C}$ and $36^{\circ} \mathrm{C}$, and for the

349 perturbations location kept fixed at a distance of $8 \mathrm{~mm}$ from the initial location

350 of the spiral wave core center. With the exception of perturbation

351 temperatures of $20^{\circ} \mathrm{C}$ and $36^{\circ} \mathrm{C}$, for which no attraction occurred, rotors were

352 attracted to the cooler perturbation following a three-phase pattern: 1) a slow

353 transient phase in which rotors were first slowly drifting, 2) a fast transient

354 phase in which the rotors were rapidly drifting towards the perturbation, and 3) 
355 a steady state phase where the rotors anchored around the perturbation. For 356 perturbation temperatures of $28^{\circ} \mathrm{C}$ or higher, the slow transient phase 1 was 357 relatively short, and its duration was found to decrease as the perturbation 358 temperature decreased. An example is given in Fig. $6 \mathrm{~B}$ for $\mathrm{T}_{2}=28^{\circ} \mathrm{C}$ (the 359 results for all other temperatures are given in Fig. S2). The left panel shows 360 the tip trajectory (in green) and the mean trajectory (in black), while the right 361 panel shows the distance (and mean distance, in black) of the spiral wave tip 362 trajectory from the center of perturbation that is located at $(x, y)=(15,23 \mathrm{~mm})$ 363 as a function of the first 20 seconds of activity, with the three characteristic 364 phases marked. For temperatures lower than $28^{\circ} \mathrm{C}$, the slow transient phase 365 was long, characterized by a first detraction and then attraction to the 366 perturbation. Moreover, in contrast to the higher temperatures, the duration of 367 the transient phase increased as the perturbation temperature decreased. An 368 example for this pattern is given in Fig. $6 \mathrm{C}$ for the case of $\mathrm{T}_{2}=26^{\circ} \mathrm{C}$, Similarly to 369 Fig. 6B. For $\mathrm{T} 2=36^{\circ} \mathrm{C}$ and $\mathrm{T} 2=20^{\circ} \mathrm{C}$ no attraction for the RTP occurred. At the $370 \mathrm{~T} 2=36^{\circ} \mathrm{C}$ case the spiral wave diverted awhile, and returned to the middle of 371 the tissue, without attracting the RTP, due to the small gradient in the 372 heterogeneity of excitability. On the other hand, the rotor trajectory at the $373 \mathrm{~T} 2=20^{\circ} \mathrm{C}$ case is having a similar pattern to the $22^{\circ} \mathrm{C}-26^{\circ} \mathrm{C}$ cases, which 374 include a long slow transient. However, for the $\mathrm{T} 2=20^{\circ} \mathrm{C}$ no attraction 375 established in the time frame of the simulation (50sec).

377 To better quantify the quality of rotor attraction to the local temperature 378 perturbation, an attraction cost function, $\phi$, was established that considers both speed of attraction and its steady state properties. Referring to the right 380 panel of Fig. 6B-C we defined STI [ms] as the slow transient interval, and $M D$ 381 [mm] as the mean distance of the spiral wave from the perturbation center at 382 steady state. To calculate the STI, the time derivative of the mean tip distance 383 (i.e., the gradient of the black curve in Fig. 6B, right) was first calculated, and 384 the STI was defined as the time corresponding to the maximal derivative. 385 These parameters were calculated for all perturbation temperatures, and in 386 order to account for the mixed units, each was normalized to obtain a mean of 
38710 with a standard deviation of 3 , resulting in corresponding $\overline{S T I}$ and $\overline{M D}$. The

388 cost function was calculated as the following root-mean-square:

$$
\phi=\sqrt{\overline{S T I}^{2}+\overline{M D}^{2}} \text {. }
$$

390 Figure $7 \mathrm{~A}$ shows $S T I$ and $M D$ as a function of the perturbation temperature, 391 while panel B shows the cost function values, clearly demonstrating an 392 optimal perturbation temperature of $28^{\circ} \mathrm{C}$. At that temperature the optimal 393 balance between fast drifting time to the perturbation and anchoring at a low 394 distance from it was reached.

395 In order to check if the optimal temperature changes for perturbations at other 396 pre-set locations, we conducted simulations with varying temperature 397 perturbations using additional distances of 6,7 and $9 \mathrm{~mm}$ (which was the 398 maximal distance that enabled attraction at $28^{\circ} \mathrm{C}$ ). The optimal temperature of $39928^{\circ} \mathrm{C}$ was obtained in all these pre-set distances, similarly to the 8-mm case. 400 Hence, we can infer that the optimal temperature that yields fast and stable 401 drifting is $28^{\circ} \mathrm{C}$ regardless of the pre-set distance of the perturbation from the 402 rotor, and therefore was used in the rest of our simulation study.

\section{Tissue Simulations - Local Perturbations at Varying Locations}

405 In the previous section we found that a local perturbation with a temperature 406 of $\mathrm{T}_{2}=28^{\circ} \mathrm{C}$ yielded the optimal performance in terms of rotor attraction quality. 407 Here we model such a perturbation, while we vary its location to study the 408 quality of rotor attraction as a function of the initial distance, $d[\mathrm{~mm}]$, between 409 the perturbation and the core of the spiral wave. The mean tip trajectories 410 during 15 seconds of activity are shown in Fig. $8 \mathrm{~A}$ for $d$ between 5 and $11 \mathrm{~mm}$, 411 while the full trajectories as well as their corresponding distance from the 412 perturbation center as a function of time are given in Fig. S3. Rotor attraction 413 was successful only at or below a critical distance of $d_{c r}=9 \mathrm{~mm}$. Over this 414 threshold, no significant effect of the perturbation on rotor meandering or 415 drifting was observed. As expected, when attraction was successful, it was 416 faster as $d$ was smaller. The slow transient interval (STI) was calculated for 417 each case as in the previous section and plotted against $d$ in Fig. 8B. The STI 418 showed an exponentially decreasing dependence on $d$, following the following 419 best-fitted relationship $\left(R^{2}=0.9995\right)$, 
421 Following this relationship, the attraction of a rotor at an initial distance of

$$
S T I=2.16 \cdot 10^{-3} \times e^{1.735 d} .
$$
$\mathrm{d}=10 \mathrm{~mm}$ is expected to require a perturbation application for more than 70 sec, which seems to be clinically impractical.

\section{DISCUSSION}

In this study, the effects of externally applied spatial temperature gradients on basic electrophysiological properties of a single atrial myocyte and on spiral wave dynamics in the atrial tissue were numerically studied using a biophysical model of a human atrial tissue. Ion channel remodeling corresponding to chronic AF conditions were employed. Our main findings are as follows: 1) in a single cell with incorporated AF remodeling, temperature exerts a significant effect on recovery time from activation, such that recovery time increases exponentially with decreasing temperatures. In contrast, APD is not affected by temperature in AF conditions; 2) when a linear temperature gradient is applied to the tissue, existing spiral waves drift in a predictable pattern, with one velocity vector component that is parallel and proportional to the negative of the temperature gradient and the other component depends on the rotation direction of the spiral; overall - spirals drift towards the colder region in the tissue, where excitability (as indicated by the sodium channel availability) obtains a global minimum; and 3) a local small temperature perturbation can be employed to attract and anchor a distant spiral wave. The feasibility and quality of such attraction depends on both the temperature of the perturbed tissue as well as on the initial distance between the perturbation and the center of the spiral wave.

Previous experimental and numerical studies have shown that spiral waves can drift in the existence of diffusivity gradient, when the shape of the AP is spatially uniform [42], or in the presence of ionic heterogeneities toward regions with reduced excitability, where an APD gradient is established. Ten Tusscher and Panfilov [27] have shown using a ventricular numerical model that in the presence of a spatial gradient in the APD due to local variations in 
450 the $\mathrm{K}+$ currents, spiral waves drifted toward regions of longer spiral wave 451 period. In accordance with our study, the drift velocity consisted of a 452 component along the gradient that was proportional to its magnitude, and a 453 perpendicular component with a direction that depended on the direction of 454 the spiral wave rotation. In line with these results, Calvo et. al. [32] 455 demonstrated that spatial heterogeneity of IK1 channel distribution may cause 456 rotors to drift in the pulmonary vein - left atrial junction (PV-LAJ). This effect 457 was attributed to a gradient in excitability near the rotor pivoting point. Still, 458 both these studies as well as other studies [30-31] were motivated by inherent 459 electrophysiological gradients in the cardiac tissue that may potentially initiate 460 or alter the dynamics of arrhythmias. To the best of our knowledge, the idea to 461 exploit the notion that rotors drift towards low excitability regions in order to 462 artificially attract and anchor rotors for potential ablation applications is novel. 463 However, artificially inducing gradients in the ion channel densities is not 464 feasible clinically. In contrast, imposing local temperature perturbation to the 465 tissue via an inserted catheter is simple. Since temperature sustains a 466 significant effect on the rate constant of biological reactions, it can be used to 467 modulate cellular electrophysiological properties. Indeed, our results show 468 that temperature variations had a substantial effect on the cellular recovery 469 time in both normal and chronic AF remodeled cells (Fig. 2). Noteworthy, 470 while temperature also had a significant effect on the APD in normal cells, 471 such effect was negligible for the AF remodeled cells. Nevertheless, we found 472 that the large sensitivity of the recovery time due to temperature variations 473 was sufficient to induce strong enough spatial gradients of excitability and 474 induce spiral wave drifting in the 2D models.

475 Some experimental support to our results, indicating that rotor drifting can be 476 induced by a local temperature gradient, can be found in a recent study by 477 Yamazaki et al. [26]. In their experiments, conducted on the left ventricle of 478 the rabbit heart, rotors that were underlying sustained ventricular tachycardia 479 were transformed by regional cooling from stationary to nonstationary when 480 regional cooling was applied. The temperature in the target area was $481 \sim 30.1^{\circ} \mathrm{C} \pm 1.3^{\circ} \mathrm{C}$ using a large cooling device with a diameter of $10 \mathrm{~mm}$. The 482 authors found that the regional cooling created local long refractoriness due to 483 the prolongation of the APD and the reduction of conduction velocity. Thus, 
484 cooling was demonstrated to be capable of unpinning the rotors, drift along 485 the periphery of the regional cooling region, and consequently facilitate 486 termination of reentrant activity by collisions with the boundaries. Our 487 simulations using local temperature perturbations generally support these 488 findings (Fig. 6), however without the rotor termination, apparently since the 489 RTP parameters in the simulations were not similar to those of the 490 experiments. Therefore, we tried to reproduce the experiment protocol by 491 applying RTP with $\mathrm{T} 2=30^{\circ} \mathrm{C}$ and $\mathrm{d}=9 \mathrm{~mm}$ (which is the most distant location 492 that can cause a drift for this type of RTP, as have been found in the study). 493 By applying these RTP protocol we succeeded to imitate the same behavior, 494 so the rotor had been destabilized, drifted toward the colder region, and 495 eventually terminated as a consequence of collision with the upper border 496 (Fig. 9). Also, in contrast to the experiments, we show that spiral wave drift is 497 obtained even in the AF remodeled tissue, where temperature variation 498 sustains no significant effect on the APD but only on the recovery time. Also, 499 our model revealed that the tip trajectory velocity was slower as the 500 temperature decreased, and its rotation period per cycle increased (Fig. S1). 501 These findings, along with the net drift demonstration in Fig. 5, are correlate to 502 the notion that lower temperature regions yielding a gradient in the tissue 503 excitability, and therefore slow the rotor motion and require a longer drifting 504 distance before the excitability level permits the rotor to reenter back. We 505 further found that an optimal effect in terms of spiral wave attraction time (STI, 506 slow transient interval) and the mean distance of the anchored spiral to the 507 perturbation center (MD, mean distance) can be obtained for a temperature of $50828^{\circ} \mathrm{C}$ (Fig. 7). While MD decreased monotonically with decreasing 509 perturbation temperatures (Fig. 7A), the STI was non-monotonous, and 510 obtained a minimum at $28^{\circ} \mathrm{C}$, thus defining the optimal perturbation 511 temperature. As we showed in Fig. 3 for linear temperature gradients, drifting 512 velocity was proportional to the spatial temperature gradient. Moreover, in 513 accordance with our hypothesis, the underlying ionic mechanism was linked 514 to the spatial gradient in the tissue excitability due to the temperature gradient 515 (Fig. 4). To mechanistically understand our observation that a local $28^{\circ} \mathrm{C}$ 516 temperature perturbation provides the optimal, shortest drifting time (or STI) 517 we plotted in Fig. 10A the time-averaged sodium channel availability (mean 
$518 h \times j$, averaged during a single spiral wave rotation) along the red dashed line 519 marked in Fig. 6B (left) for all the simulations described in Fig. 6. This line 520 connects between the initial center of the spiral wave and the center of 521 perturbation and thus represents an optimal drifting path for the spiral wave. 522 As Fig. 10A shows, the mean $h \times j$ profile is characterized by a local trough at $523 \mathrm{y} \sim 15 \mathrm{~mm}$ that corresponds to the low-excitability spiral wave core center, and 524 a region of low $h \times j$ values between $y=22 \mathrm{~mm}$ and $\mathrm{y}=24 \mathrm{~mm}$ that corresponds to 525 the location of the perturbation. The dashed black lines in Fig. 10A mark the 526 region between $\mathrm{y}=17 \mathrm{~mm}$ - the right peak to the right of the trough (or the edge 527 of the spiral wave core) - and $y=22 \mathrm{~mm}$ - the left edge of the perturbation. That 528 region contains the spatial gradient of $h \times j$ that is affecting the spiral wave 529 drifting. For each temperature, the mean $h \times j$ gradient in that region was 530 estimated by the slope of the best fit line between those two dashed lines. As 531 shown in Fig. 10B, the drifting time (STI) was proportional to the negative of 532 the $h \times j$ gradient. A linear regression analysis (Fig. 10C) showed indeed that 533 these two properties were linearly correlated with the following relationship $534\left(R^{2}=0.83\right)$,

$$
S T I=-12.34 \nabla(h \times j)+123 .
$$

536 Thus, for gradually decreasing perturbation temperatures the gradient of 537 excitability as sensed by the spiral wave initially slowly increased, reaching a 538 plateau at $\mathrm{T} 2=28^{\circ} \mathrm{C}$. Below that temperature, the gradient magnitude 539 decreased rapidly. As a result, the drifting time, represented by the STI, which

540 is correlated to the excitability gradient by (9), rapidly increased, deteriorating 541 the quality of attraction.

\section{$542 \quad$ Limitations}

543 In this work, the CRN model was employed with AF modifications in an 544 isotropic, electrophysiologically uniform 2D tissue to yield a single rotor 545 activity. Other AF models, e.g., the model employed by Jacquemet et al [43], 546 integrated anatomically and dynamically complex elements like 3D geometry, 547 tissue anisotropy and other physiological heterogeneities that yielded complex 548 AF conduction patterns such as multiple wavelets, spiral wave breakups and 549 collisions. Yet, the aim of our study was to establish the most fundamental 550 theory underlying the relationship between temperature gradients and rotor 
551 drifting. Thus, it was in fact our intention to employ a simplified, isotropic

552 anatomy model wherein a single rotor is active in order to allow a direct 553 analysis of such relationships, while avoiding interfering factors that will 554 render such analysis too complex. In future studies we will utilize the 555 mechanisms established in this paper to further analyze the effects of 556 temperature heterogeneities in a more complex anatomy and AF electrical 557 propagation patterns. It should also be noted that while a single rotor is 558 indeed a relatively simple kind of propagation dynamics during AF, the 559 "mother rotor" hypothesis is one of the potential suggested mechanism 560 sustaining AF, that supported by the exhibition in the AF driving region of 561 maximum dominant frequency [44]. Therefore, simulating persistent AF driver 562 as a single rotor may not be a simplified scenario, but rather a likely one.

\section{Potential clinical implications and future studies}

565 Our study aims at understanding the basic mechanisms of spiral wave drifting 566 and attraction in the presence of applied temperature perturbations, and the 567 presented results cannot be directly translated into the clinical setup. Still, this 568 study was motivated by the potential prospective clinical application of the 569 proposed method, and it is our hope that the mechanistic insights from our 570 study may be utilized in the future as a proof-of-concept in the design of a 571 new methodology for AF characterization and termination. Surely such 572 application should be comprehensively investigated in future experimental 573 and clinical studies. We believe that the interaction between rotors and 574 temperature gradients can be utilized to help tracking rotors. For example, it is 575 well-known that rotor drifting leading to Doppler-induced differences in local 576 activation periods along the direction of drift [45]. Therefore, it is hypothesized 577 that the application of local temperature perturbation while measuring the 578 electrical activity at several few pre-set locations may allow to back-track the 579 origin of a drifting rotor. This can be theoretically achieved by measuring the 580 Doppler shifts at the various locations, which arise only due to the rotor 581 drifting rather than stable rotor or ectopic source, and applying an inverse 582 reconstruction algorithm. However, causing for a controlled spiral wave drift in 583 the clinic is on the verge of impracticality, and the method presented in this 
584 study can provide a novel solution for it. The development of such an 585 algorithm, in different scenarios, is the goal of our future research. A probable 586 clinical scenario would be the application of temperature gradients in a fibrotic 587 atrial tissue. As rotors tend to stabilize around areas of fibrotic tissue or tissue 588 heterogeneities, a question arises whether and under what configurations, 589 reentrant waves can or cannot detach from fibrotic patches due to applied 590 temperature perturbations. Such a question should be undoubtedly 591 investigated in the future. Finally, future research should have to investigate if 592 application of local cooling in persistent AF patients and using the Doppler 593 Effect will enable to characterize the type of driving arrhythmogenic source, 594 being ectopic foci (and thus not being affected by the applied temperature 595 gradients and therefore not drifting) or a rotor that is expected to drift in the 596 presence of temperature gradient. We predict that a controlled spiral wave 597 drift will occur under spatial temperature gradient, causing to Doppler Effect 598 that can be used for analyze the arrhythmogenic source type. This interesting 599 classification problem should be also studied in order to fully understand the 600 limitations and potential of local temperature application in the 601 characterization of AF activity.

602

603 Competing interests: None declared

604 Funding: None

605 Ethical approval: Not required

606

\section{REFERENCES}

608 [1] Nattel S. New ideas about atrial fibrillation 50 years on. Nature 2002; 609 415(6868):219-226.

610 [2] Pandit SV, Jalife J. Rotors and the dynamics of cardiac fibrillation. Circ 611 Res 2013. 112(5):849-862.

[3] Baher A, Qu Z, Hayatdavoudi A, Lamp ST, Yang MJ, Xie F, Weiss JN. 614 Short-term cardiac memory and mother rotor fibrillation. Am J Physiol Heart C 2007. 292(1):H180-H189.

[4] Nattel S. Experimental evidence for proarrhythmic mechanisms of antiarrhythmic drugs. Cardiovasc Res 1998. 37:567-577. 
617 [5] Baumert M, Sanders P, Ganesan A. Quantitative-electrogram-based

618

619

620

621

622

623

624

625

626

627

628

629

630

631

632

633

634

635

636

637

638

639

640

641

642

643

644

645

646

647

648

649

650

651

652

653

654

655

656

657

658

659 methods for guiding catheter ablation in atrial fibrillation. Proc. IEEE. 2016. 104(2): 416-431.

[6] Ravelli F, Masè M. Computational mapping in atrial fibrillation: how the integration of signal-derived maps may guide the localization of critical sources. Europace 2014. 16(5): 714-723.

[7] Nademanee K, Lockwood E, Oketani N, Gidney B. Catheter ablation of atrial fibrillation guided by complex fractionated atrial electrogram mapping of atrial fibrillation substrate. J Cardiol 2010. 55(1):1-12.

[8] Li WJ, Bai YY, Zhang HY, Tang RB, Miao CL, Sang CH, Ma CS. Additional ablation of complex fractionated atrial electrograms after pulmonary vein isolation in patients with atrial fibrillation a metaanalysis. Circ Arrhythm Electrophysiol 2011. 4:143-148.

[9] Atienza F, Almendral J, Jalife J, Zlochiver S, Ploutz-Snyder R, Torrecilla EG, Berenfeld $O$. Real-time dominant frequency mapping and ablation of dominant frequency sites in atrial fibrillation with left-toright frequency gradients predicts long-term maintenance of sinus rhythm. Heart Rhythm 2009. 6(1):33-40.

[10] Verma A, Lakkireddy D, Wulffhart Z, Pillarisetti J, Farina D, Beardsall M, Khaykin Y. Relationship between complex fractionated electrograms (CFE) and dominant frequency (DF) sites and prospective assessment of adding DF-guided ablation to pulmonary vein isolation in persistent atrial fibrillation (AF). J. Cardiovasc Electr 2011. 22(Cl):1309-1316.

[11] Annoni MA, Arunachalam SP, Kapa S, Mulpuru S, Friedman P, Tolkachova E. Novel Quantitative Analytical Approaches for Rotor Identification and Associated Implications for Mapping. IEEE Trans Biomed Eng 2018. 65(2): 273-281.

[12] Greisas A, Zafrir Z, Zlochiver S. Detection of Abnormal Cardiac Activity Using Principal Component Analysis-A Theoretical Study. IEEE T Bio Med Eng 2015. 62(1):154-164.

[13] Arunachalam SP, Mulpuru SK, Friedman PA, Tolkacheva EG. Feasibility of visualizing higher regions of Shannon entropy in atrial fibrillation patients. Conf. Proc. IEEE Eng Med Biol Soc 2015. 44994502.

[14] Kang TL, Sébastien K, Wright M, Haissaguerre M. Atrial substrate ablation in atrial fibrillation. In: Jalife J, Zipes DP, editors. Cardiac Electrophysiology: from cell to bedside vol 5, Saunders Elsevier; 2009, p. 1059-1070.

[15] Hodgkin BA, Katz B. The effect of temperature on the electrical activity of the giant axon of the squid. J Physiol 1949. 109 (1-2):240-249.

[16] Kiyosue T, Arita M. Ionic mechanisms of action potential prolongation at low temperature in guinea-pig ventricular myocytes. J Physiol 1993. 468:85-106. 
660 [17] Zhao Y, Boulant J. Temperature effects on neuronal membrane

661

662

663

664

665

666

667

668

669

670

671

672

673

674

675

676

677

678

679

680

681

682

683

684

685

686

687

688

689

690

691

692

693

694

695

696

697

698

699

700

701

702

703 potentials and inward currents in rat hypothalamic tissue slices. $\mathrm{J}$ Physiol 2005. 564(1):245-57.

[18] Plonsey R, Barr RC. Beyond $\mathrm{H}-\mathrm{H}$ models: Temperature, in Bioelectricity - A quantitative approach, 3rd ed. 2007. p. 139-142.

[19] Bjørnstad H, Tande PM, Lathrop DA, Refsum H. Effects of temperature on cycle length dependent changes and restitution of action potential duration in guinea pig ventricular muscle. Cardiovasc Res 1993. 27(6):946-950.

[20] Dumaine R, Towbin JA, Brugada P, Vatta M, Nesterenko DV, Nesterenko VV, Antzelevitch C. Ionic Mechanisms Responsible for the Electrocardiographic Phenotype of the Brugada Syndrome Are Temperature Dependent. Circ Res 1999. 85(9):803-809.

[21] Freysz M, Timour Q, Mazze RI, Bertrix L, Cohen S, Samii K, Faucon G. Potentiation by mild hypothermia of ventricular conduction disturbances and reentrant arrhythmias induced by bupivacaine in dogs. Anesthesiology 1989. 70:799-804.

[22] Kochs E. Electrophysiological monitoring and mild hypothermia. J Neurosurg Anesth 1995. 7(3):222-228.

[23] Rankin AC, Rae P. Cardiac arrhythmias during rewarming of patients with accidental hypothermia. $\mathrm{Br}$ Med J (Clin. Res. Ed) 1984. 289(6449):874-877.

[24] Fenton FH, Gizzi A, Cherubini C, Pomella N, Filippi S. Role of temperature on nonlinear cardiac dynamics. Phys Rev E 2013. 87(4): 042717.

[25] Filippi S, Gizzi A, Cherubini C, Luther S, Fenton FH. Mechanistic insights into hypothermic ventricular fibrillation: the role of temperature and tissue size. Europace 2014. 16(3):424-434.

[26] Yamazaki M, Honjo H, Ashihara T, Harada M, Sakuma I, Nakazawa K, Kamiya K. Regional cooling facilitates termination of spiral-wave reentry through unpinning of rotors in rabbit hearts. Heart Rhythm 2012. 9(1):107-114.

[27] Ten Tusscher KHWJ, Panfilov AV. Reentry in heterogeneous cardiac tissue described by the Luo-Rudy ventricular action potential model. Am J Physiol-Heart C 2003. 284(2):H542-H548.

[28] Xie F, Qu Z. Electrophysiological heterogeneity and stability of reentry in simulated cardiac tissue. Am J Physiol Heary C. 2001. 280: 535545.

[29] Antzelevitch C. Cardiac heterogeneity Electrical heterogeneity within the ventricular wall. Basic Res Cardiol 2001. 96:517-527.

[30] Pandit SV, Berenfeld O, Anumonwo JM, Zaritski RM, Kneller J, Nattel $\mathrm{S}$, Jalife $\mathrm{J}$. Ionic determinants of functional reentry in a 2-D model of human atrial cells during simulated chronic atrial fibrillation. Biophys $\mathrm{J}$ 2005. 88(6): 3806-3821. 
704

705

706

707

708

709

710

711

712

713

714

715

716

717

718

719

720

721

722

723

724

725

726

727

728

729

730

731

732

733

734

735

736

737

738

739

740

741

742

743

744

745
[31] Jalife J, Gray R. Drifting vortices of electrical waves underlie ventricular fibrillation in the rabbit heart. Acta Physiol Scand 1996. 157(2):123-131.

[32] Calvo CJ, Deo M, Zlochiver S, Millet J, Berenfeld O. Attraction of rotors to the pulmonary veins in paroxysmal atrial fibrillation: a modeling study. Biophys J 2014. 106(8):1811-1821.

[33] Courtemanche M, Ramirez RJ, Nattel S. Ionic mechanisms underlying human atrial action potential properties: insights from a mathematical model. Am J Physiol 1998. 275(1):H301-H321.

[34] Courtemanche M, Ramirez RJ, Nattel S. Ionic targets for drug therapy and atrial fibrillation-induced electrical remodeling: insights from a mathematical model. Cardiovasc Res 1999. 42(2):477-489.

[35] Sakakibara Y, Wasserstrom JA, Furukawa T, Jia H, Arentzen CE, Hartz RS, Singer DH. Characterization of the sodium current in single human atrial myocytes. Circ Res 1992. 71:535-546.

[36] Wang Z, Fermini B, Nattel S. Sustained depolarization-induced outward current in human atrial myocytes. Evidence for a novel delayed rectifier K+ current similar to Kv1.5 cloned channel currents. Circ Res 1993. 73(6):1061-1076.

[37] Mazhari R, Greenstein JL, Winslow RL, Marbán E, Nuss HB. Molecular Interactions between Two Long-QT Syndrome Gene Products, HERG and KCNE2, Rationalized by In Vitro and In Silico Analysis. Circ Res 2001. 89(1):33-38.

[38] Terrenoire C, Clancy CE, Cormier JW, Sampson KJ, Kass RS. Autonomic control of cardiac action potentials: role of potassium channel kinetics in response to sympathetic stimulation. Circ Res 2005. 96(5): e25-34.

[39] Ten Tusscher KHWJ, Noble D, Noble PJ, Panfilov AV. A model for human ventricular tissue. Am J Physiol Hear Circ Physiol 2004. 286(4):H1573-H1589.

[40] Paul A, Narasimhan A, Kahlen FJ, Das SK. Temperature evolution in tissues embedded with large blood vessels during photo-thermal heating. J Therm Biol 2014. 41:77-87.

[41] Nayak AR, Shajahan TK, Panfilov AV, Pandit R. Spiral-wave dynamics in a mathematical model of human ventricular tissue with myocytes and fibroblasts. PLoS One 2013. 8(9):e72950.

[42] Wellner M, Pertsov AM, Jalife J. Spiral drift and core properties. Phys Rev E Stat Phys Plasmas Fluids Relat Interdiscip Topics 1999. 59(5A): 5192-5204.

[43] Jacquemet V, Virag N, Ihara Z, Dang L, Blanc O, Zozor S, Vesin JM, Kappenberger L, Henriquez C. Study of unipolar electrogram morphology in a computer model of atrial fibrillation. J Cardiovasc Electrophysiol 2003.14(s10):S172-S179. 
746

747

748

749

750

751

[44] Narayan SM, Jalife J. CrossTalk proposal: Rotors have been demonstrated to drive human atrial fibrillation. J. Physiol., 2014. 592(15):3163-3166.

[45] Gray RA. Theory of rotors and arrhythmias. In: Jalife J, Zipes DP, editors. Cardiac Electrophysiology: From Cell to Bedside (Sixth Edition). Saunders Elsevier. 2013. 341-350. 


\section{Figure Legends:}

753

754 Figure 1 Two types of spatial temperature gradients that were 755 employed in the study. Left -a linear temperature gradient, in this example

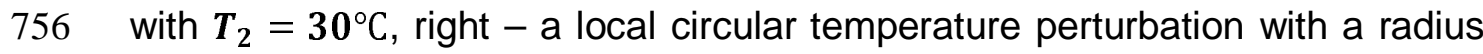
757 of $1 \mathrm{~mm}$, here with $\boldsymbol{T}_{\mathbf{2}}=30^{\circ} \mathrm{C}$

759 Figure 2 Effects of temperature dependency on single cell action 760 potential and excitability. A-B. Transmembrane voltage (top) and sodium 761 channel availability $(\mathrm{h} \times \mathrm{j})$ in time for various temperatures and for the standard 762 CRN model (A, [33]) and the AF model (B, [34]). C. Temperature dependency 763 on the model outcomes for the two kinetic models: APD 90 (top left), sodium 764 channel recovery time (bottom left) measured as the time interval between $\mathrm{h} \times \mathrm{j}$ 765 of 0.1 and 0.9 , AP amplitude (top right), and planar conduction velocity 766 (bottom right).

767

768 Figure 3 Linear temperature gradients in 2D tissue simulations. A. 769 Effects of temperature gradient sign and direction of spiral wave rotation. 770 Negative and positive gradients are marked in blue and green, respectively. 771 Clockwise and counter clockwise rotations are marked in light and dark 772 shades, respectively. B. Spatiotemporal spiral wave tip trajectory and its mean 773 (light and bold lines, respectively) for linear temperature gradients of various 774 temperatures, T2. All spiral waves rotated clockwise. C. Mean horizontal and 775 vertical drifting velocities as a function of the temperature gradient. D. The 776 angle between the drift velocity vector and the gradient vector as a function of 777 the temperature gradient.

778

779 Figure 4 Drifting occurs from high to low tissue excitability in a tissue 780 with a linear temperature gradient. A. Mean spiral wave tip drifting trajectory 781 for a model with a linear temperature gradient with $\mathrm{T} 2=30^{\circ} \mathrm{C}$ and a clockwise 782 rotating spiral wave. B-C. A time-space plot (TSP) of the sodium channel 783 availability $(\mathrm{h} \times \mathrm{j})$ along the dashed profile in panel $A$. The $\mathrm{x}$-axis is represented 784 by a normalized linear coordinate along the diagonal profile (p). A zoomed in 785 section is given in panel $\mathrm{C}$, demonstrating the average lower sodium channel 786 availability to the top-left of the spiral wave core. D. Time-averaged sodium 787 channel availability along the profile marked in panel A, showing that drifting 788 indeed occurred towards the globally minimal average $\mathrm{h} \times \mathrm{j}$. 
789 Figure $5 \quad$ Activation map of a single spiral wave rotation corresponding

790 to the linear temperature gradient simulation with $\mathrm{T} 2=30^{\circ} \mathrm{C}$. The orange and

791 green points correspond to the initial and end points of spiral wave tip during

792 this single cycle. A net drift towards the top-left corner is seen during the

793 rotation. See more details in the text.

794

795 Figure 6 Simulations of local perturbations of varying temperatures. A.

796 Left - mean tip trajectories of a spiral wave in the presence of the local

797 perturbations. Right - time-space plots of the trajectories. B-C. Detailed tip

798 trajectories and mean trajectories (in black) for the cases of $\mathrm{T} 2=28^{\circ} \mathrm{C}$ and

$799 \mathrm{~T} 2=26^{\circ} \mathrm{C}$ ( $\mathrm{B}$ and $\mathrm{C}$, respectively). Left - the distance of the mean tip

800 trajectory from the center of perturbation as a function of time. These traces

801 show the typical 3 phases of drifting - slow transient phase (Phase 1), fast

802 transient phase (Phase 2) and steady state phase (Phase 3). The measures

803 of slow transient interval (STI) and mean distance at steady state (MD) are

804 marked.

805

806 Figure $7 \quad$ Quality of spiral wave attraction as a function of perturbation 807 temperature. A. Slow transient interval (STI) and mean distance (MD) of the 808 spiral tip. B. Cost function, as defined in Eq. 7, as a function of temperature, 809 showing a clear optimal temperature of $28^{\circ} \mathrm{C}$.

810

811 Figure 8 Attraction of a spiral wave using a local perturbation of $812 \mathrm{~T} 2=28^{\circ} \mathrm{C}$ and varying locations. A. Time-space plots of the tip trajectory. $\mathrm{B}$. 813 Duration of the slow transient interval (STI) phase of attraction as a function of 814 the initial distance, $\mathrm{d}$, between the spiral wave core center and the center of 815 perturbation.

816

817 Figure 9 Employing RTP with $\mathrm{T}_{2}=30^{\circ} \mathrm{C}$ and $\mathrm{d}=9 \mathrm{~mm}$, yielding the rotor to 818 be destabilized, drift toward the colder region, and eventually terminated as a 819 consequence of collision with the upper border (at $Y=30 \mathrm{~mm}$ ).

821 Figure 10 Analysis of spiral wave drifting mechanism for a local 822 temperature perturbation. A. Mean sodium channel availability along the 823 dashed vertical line in Fig. 6B (left). The region between the two dashed black 824 lines refers to the vertical distance between the spiral wave center and the 825 perturbation center, and the gradient of excitability in that region was found to 826 correlate with the drifting time of the spiral wave before anchoring to the 827 perturbation. B. The gradient of excitability (between the two dashed lines in 828 panel A) and the STI as a function of perturbation temperature. C. 829 Relationship between the gradient of excitability and the STI reveals a 830 decreasing monotonic function. 
832 Figure S1 Spiral wave properties as function of constant tissue 833 temperature. Left column: Rotor activation frequency (f1), which is the fast 834 frequency that determines the interval between sequential local excitation 835 (top), spiral wave tip rotation frequency (f2) (middle), which is the slow 836 frequency that determines the rotation rate of a rotor, and the mean tip 837 velocity (bottom). Spiral wave dynamics exhibited a monotonic behavior as 838 function of temperature, so that the wavefront propagation is being slower and 839 have smaller frequencies with decreasing temperatures. Right column: Spiral 840 wave tip trajectory of one rotation cycle for several cases ( $T$ equal to $30^{\circ} \mathrm{C}$, $84133^{\circ} \mathrm{C}, 37^{\circ} \mathrm{C}$ and $40^{\circ} \mathrm{C}$ ), demonstrated the different trajectory patterns as 842 function of temperature.

843

844 Figure S2 Simulations of local perturbations of varying temperatures. A.

845 Tip trajectories of a spiral wave in the presence of the local perturbations. In 846 black - mean trajectories. Perturbation location is marked by a circle. B. 847 Distance of the mean tip trajectory from the center of perturbation as a 848 function of time.

849

850 Figure S3 Simulations of local perturbations of varying initial distance 851 from the spiral wave core. A. Tip trajectories of a spiral wave in the presence 852 of the local perturbations. In black - mean trajectories. Perturbation location is 853 marked by a circle. B. Distance of the mean tip trajectory from the center of 854 perturbation as a function of time.

TABLE 1

Q10 VALUES FOR EACH IONIC CURRENT

\begin{tabular}{ccc}
\hline \hline Current & $Q_{10}$ & Reference \\
\hline$I_{N a}$ & 3 & {$[33]$} \\
$I_{t o}$ & 2.2 & {$[34]$} \\
$I_{K u r}$ & 2.2 & {$[34]$} \\
$I_{K r}$ & 3.3 & {$[35]$} \\
$I_{K s}$ & 2.57 & {$[36]$} \\
$I_{C a-L}$ & 2.2 & {$[37]$} \\
\hline
\end{tabular}


855 FIGURES

856
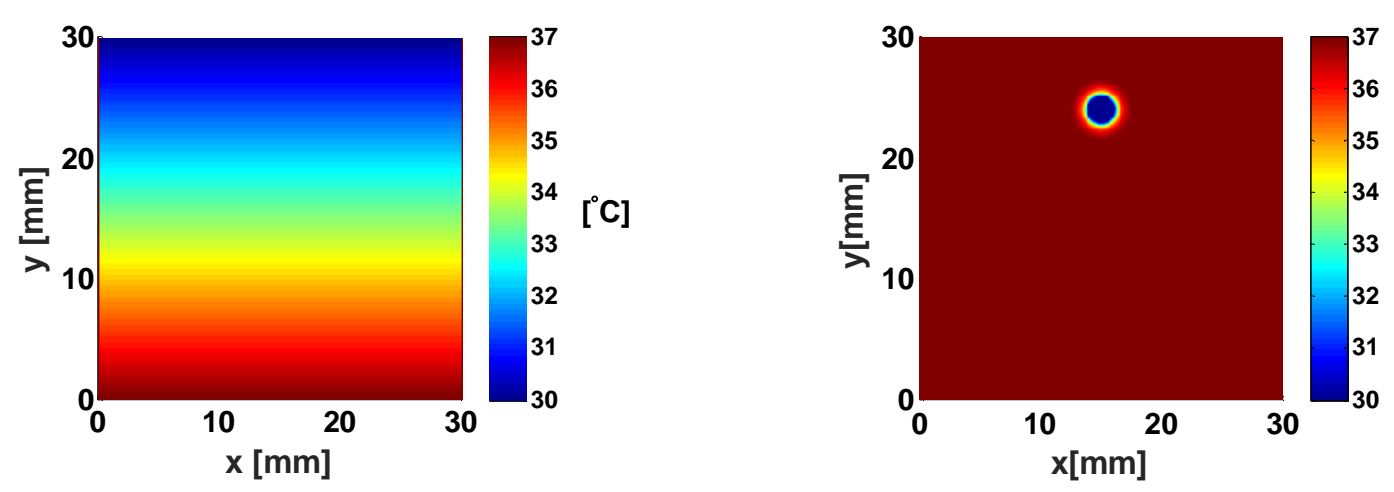

Fig. 1. Two types of spatial temperature gradients that were employed in the study. Left -a linear temperature gradient, in this example with $\boldsymbol{T}_{\mathbf{2}}=\mathbf{3 0} \mathbf{C}^{\circ} \mathrm{C}$ right - a local circular temperature perturbation with a radius of $1 \mathrm{~mm}$, here with $\boldsymbol{T}_{2}=30^{\circ} \mathrm{C}$ 

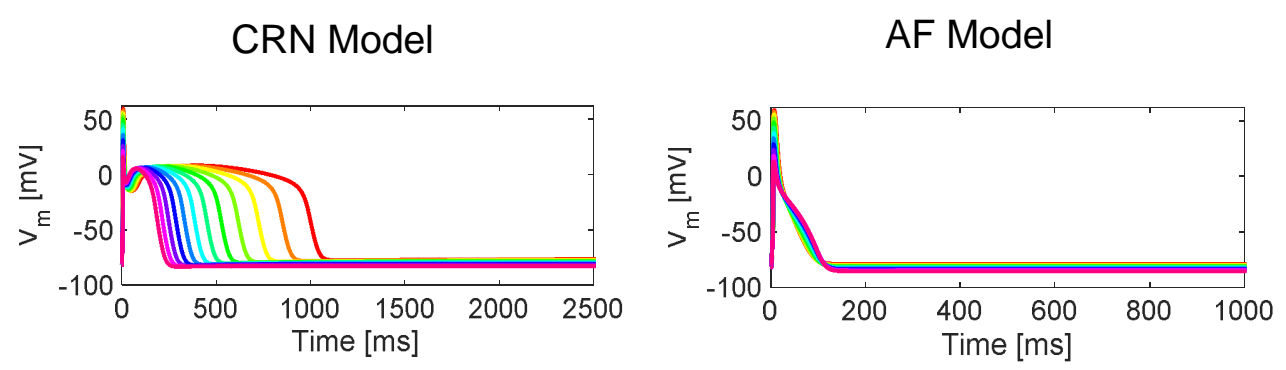

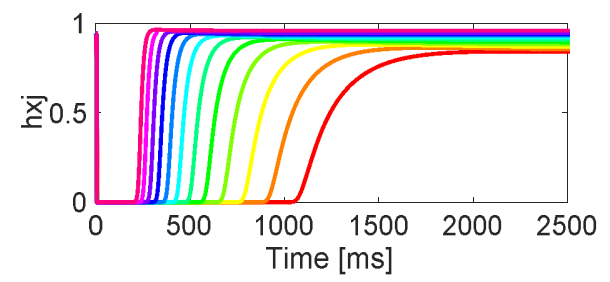

A
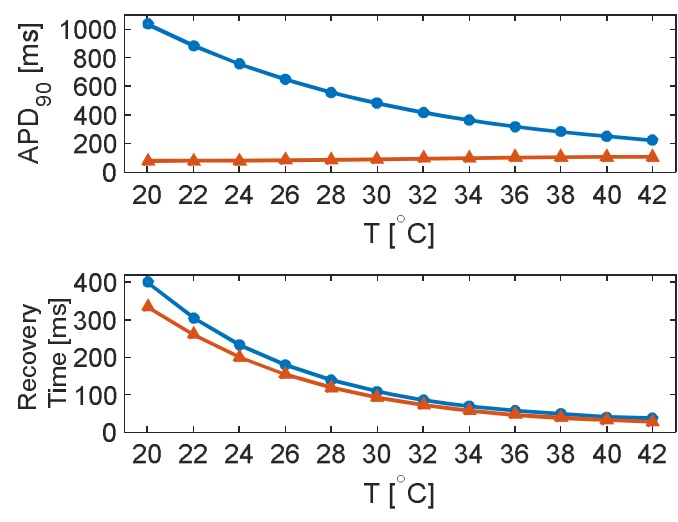

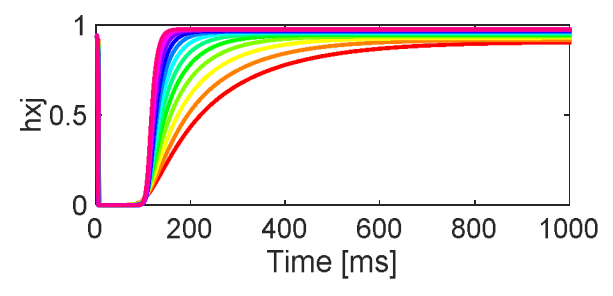

B
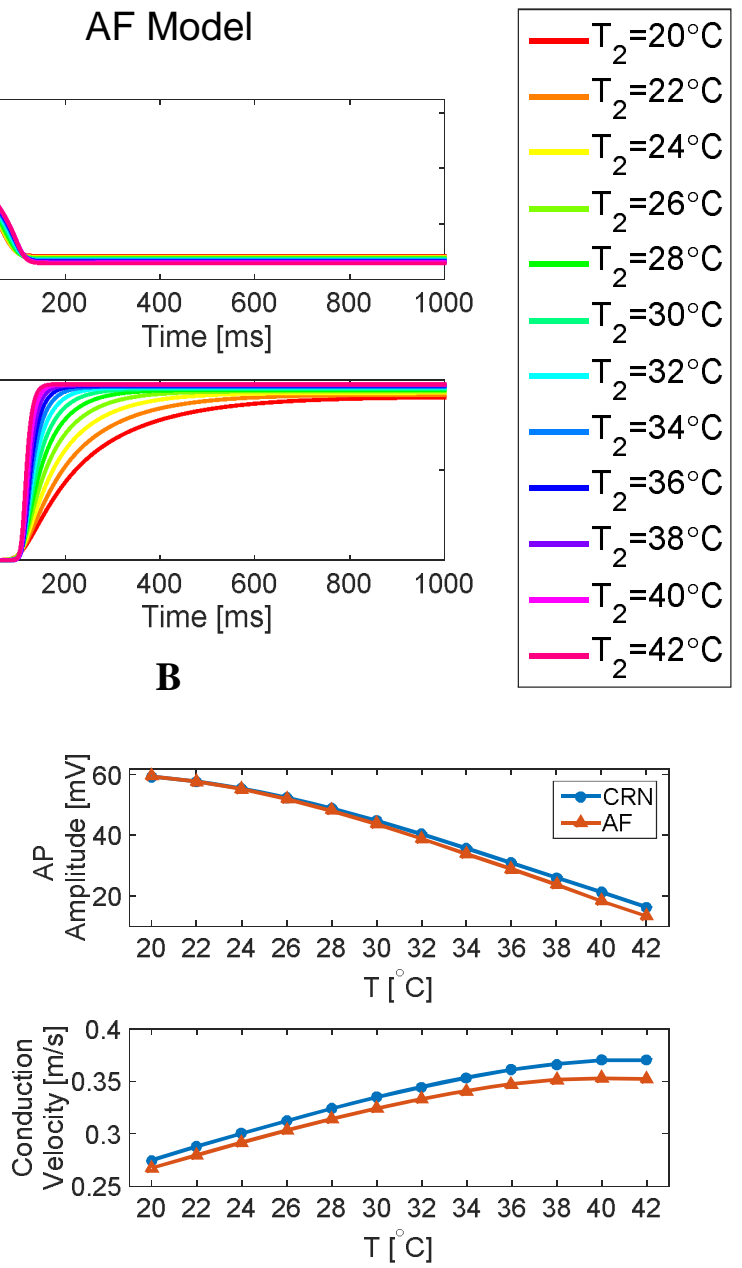

C

Fig. 2. Effects of temperature dependency on single cell action potential and excitability. A-B. Transmembrane voltage (top) and sodium channel availability $(h \times j)$ in time for various temperatures and for the standard $C R N$ model (A, [33]) and the $A F$ model ( $B$, [34]). C. Temperature dependency on the model outcomes for the two kinetic models: $A P D_{90}$ (top left), sodium channel recovery time (bottom left) measured as the time interval between $\mathrm{h} \times \mathrm{j}$ of 0.1 and 0.9 , AP amplitude (top right), and planar conduction velocity (bottom right). 


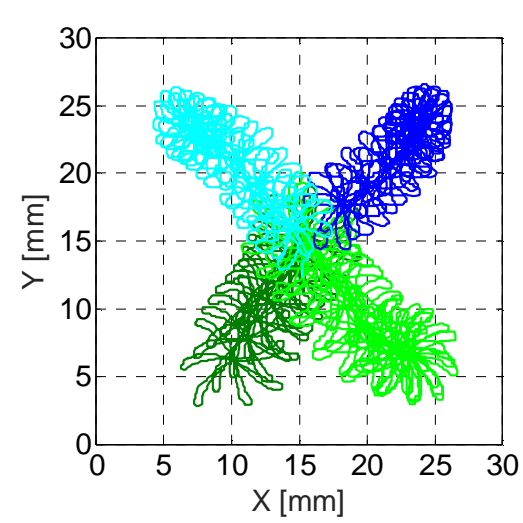

$-\mathrm{T}_{2}=40^{\circ} \mathrm{C}$ counter clock wise

$-\mathrm{T}_{2}=40^{\circ} \mathrm{C}$ clock wise

$-\mathrm{T}_{2}=34^{\circ} \mathrm{C}$ counter clock wise

$\mathrm{T}_{2}=34^{\circ} \mathrm{C}$ clock wise

A

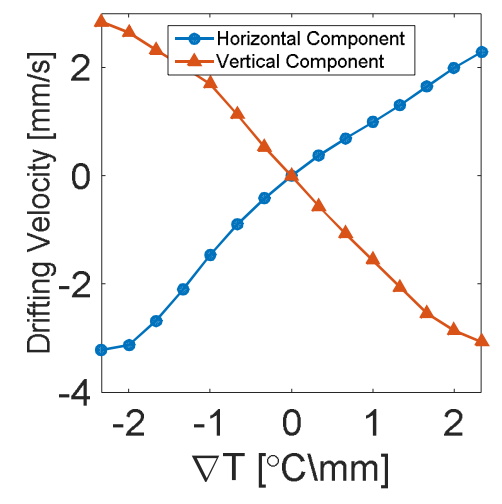

C
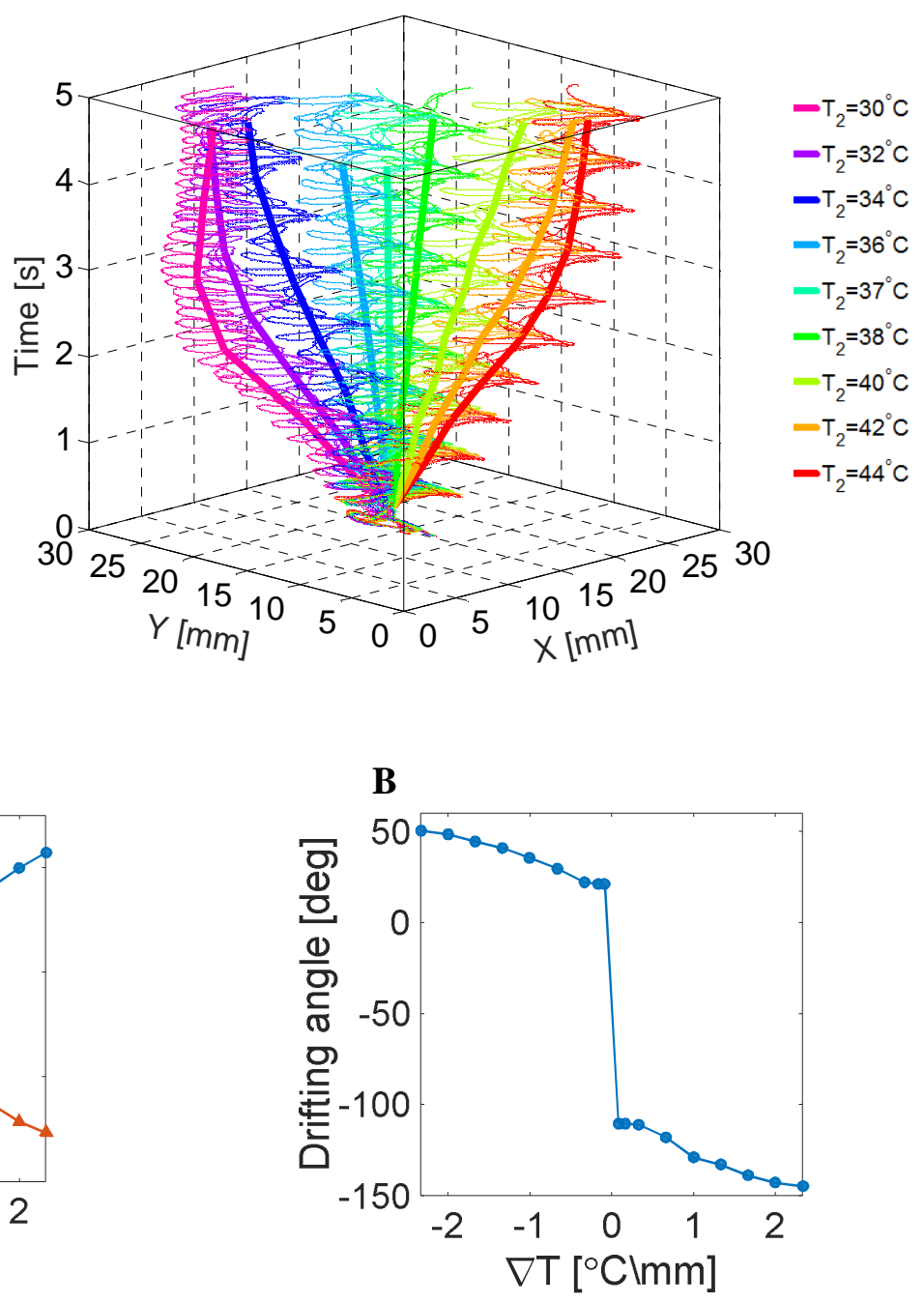

D

Fig. 3. Linear temperature gradients in 2D tissue simulations. A. Effects of temperature gradient sign and direction of spiral wave rotation. Negative and positive gradients are marked in blue and green, respectively. Clockwise and counter clockwise rotations are marked in light and dark shades, respectively. B. Spatiotemporal spiral wave tip trajectory and its mean (light and bold lines, respectively) for linear temperature gradients of various temperatures, T2. All spiral waves rotated clockwise. C. Mean horizontal and vertical drifting velocities as a function of the temperature gradient. D. The angle between the drift velocity vector and the gradient vector as a function of the temperature gradient. 


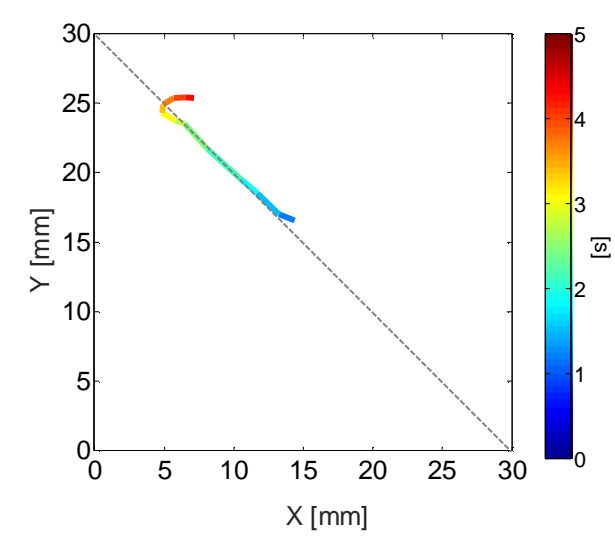

A

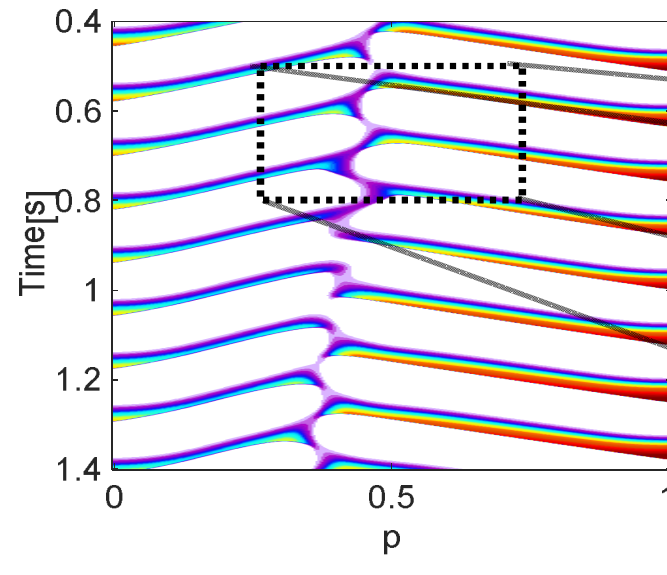

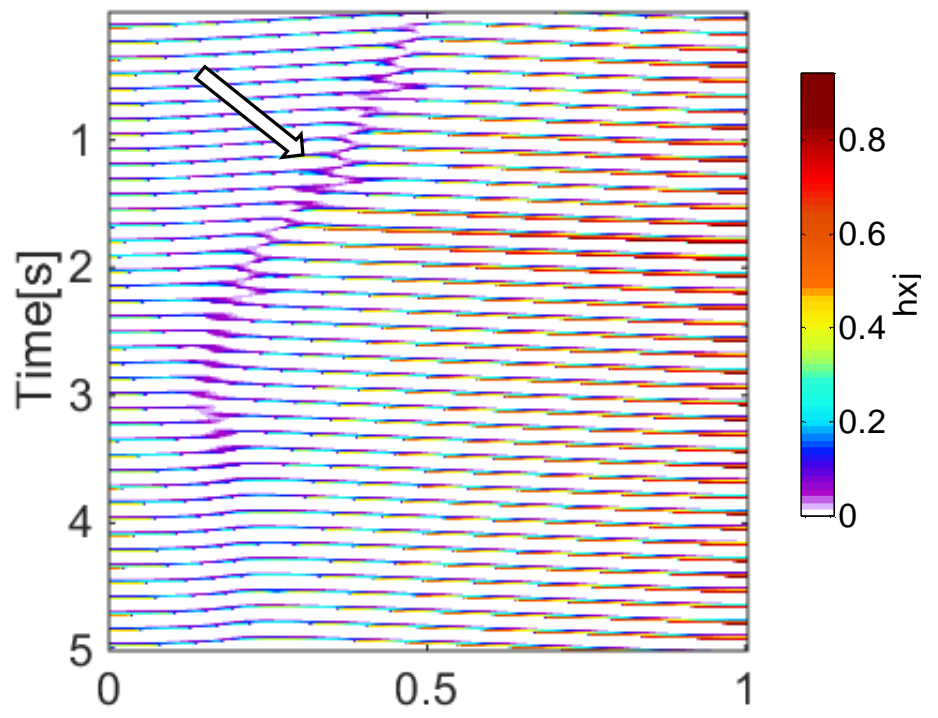

B

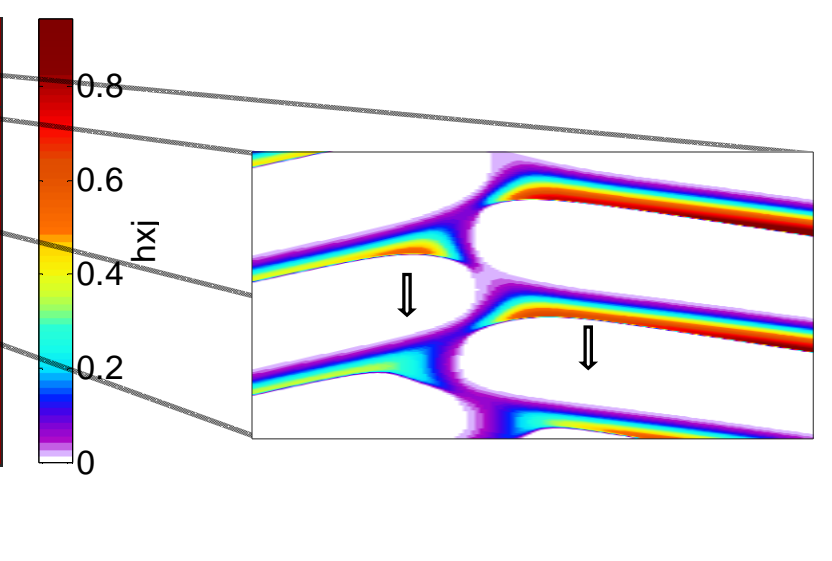

C

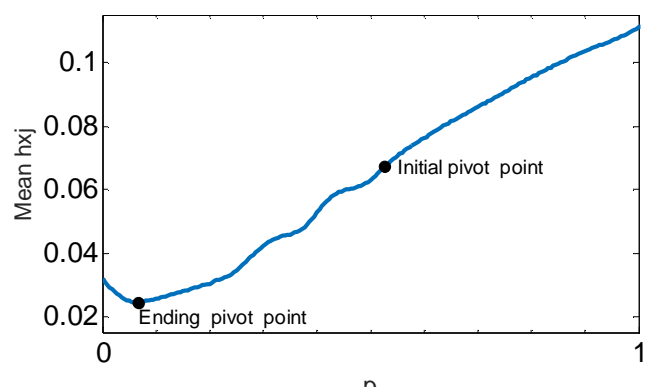

D

Fig. 4. Drifting occurs from high to low tissue excitability in a tissue with a linear temperature gradient. A. Mean spiral wave tip drifting trajectory for a model with a linear temperature gradient with $\mathrm{T} 2=30^{\circ} \mathrm{C}$ and a clockwise rotating spiral wave. B-C. A time-space plot (TSP) of the sodium channel availability $(\mathrm{h} \times \mathrm{j})$ along the dashed profile in panel $A$. The $\mathrm{x}$-axis is represented by a normalized linear coordinate along the diagonal profile (p). A zoomed in section is given in panel $\mathrm{C}$, demonstrating the average lower sodium channel availability to the top-left of the spiral wave core. D. Time-averaged sodium channel availability along the profile marked in panel $A$, showing that drifting indeed occurred towards the globally minimal average $\mathrm{h} \times \mathrm{j}$. 


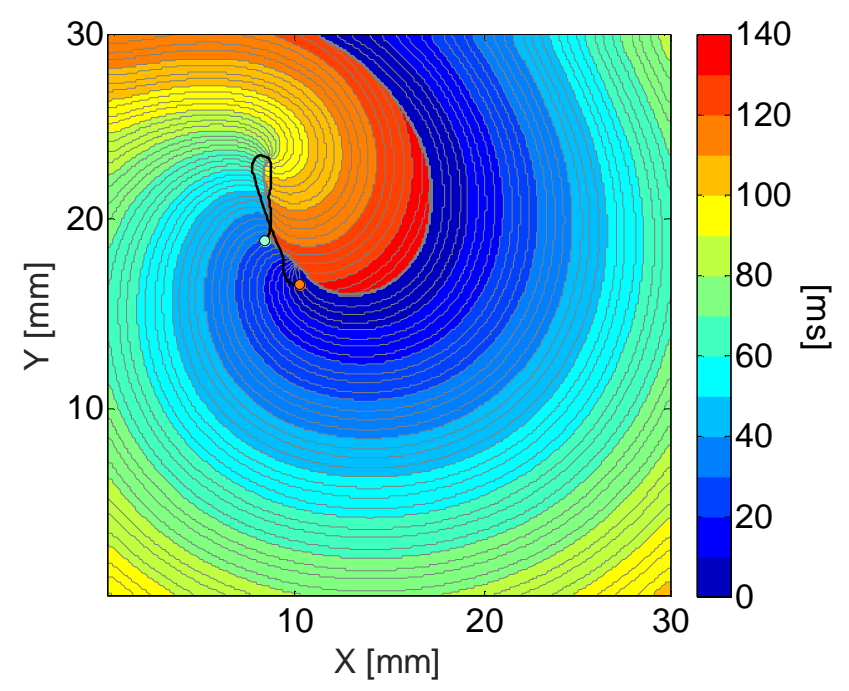

Fig. 5. Activation map of a single spiral wave rotation corresponding to the linear temperature gradient simulation with $\mathrm{T} 2=30^{\circ} \mathrm{C}$. The orange and green points correspond to the initial and end points of spiral wave tip during this single cycle. A net drift towards the top-left corner is seen during the rotation. See more details in the text. 


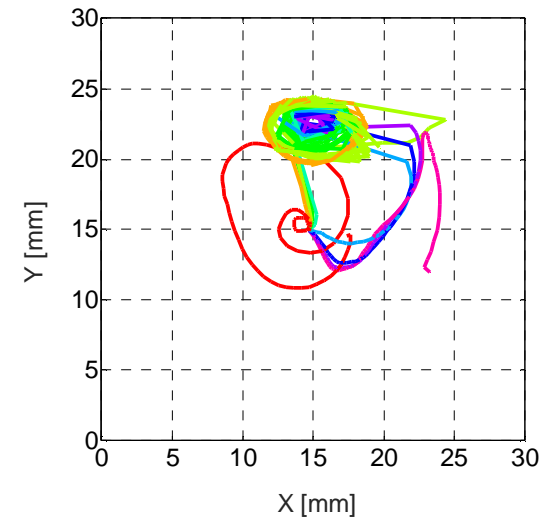

A

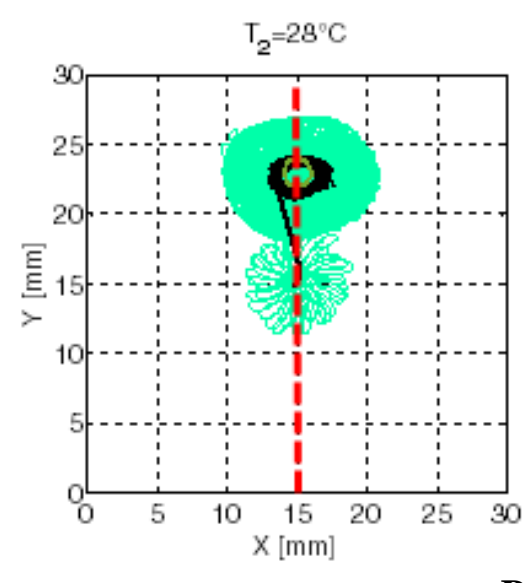

B

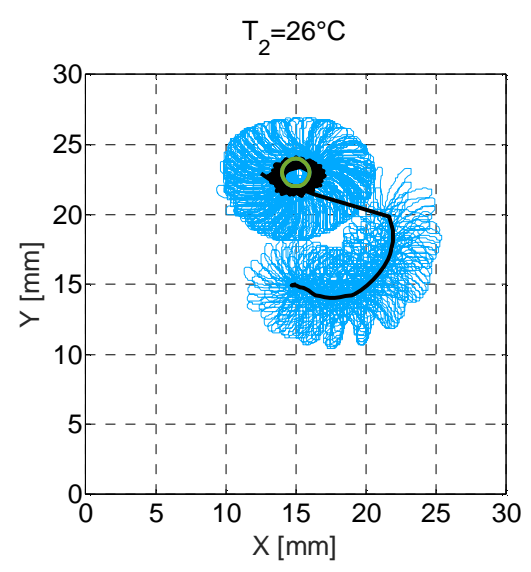

C

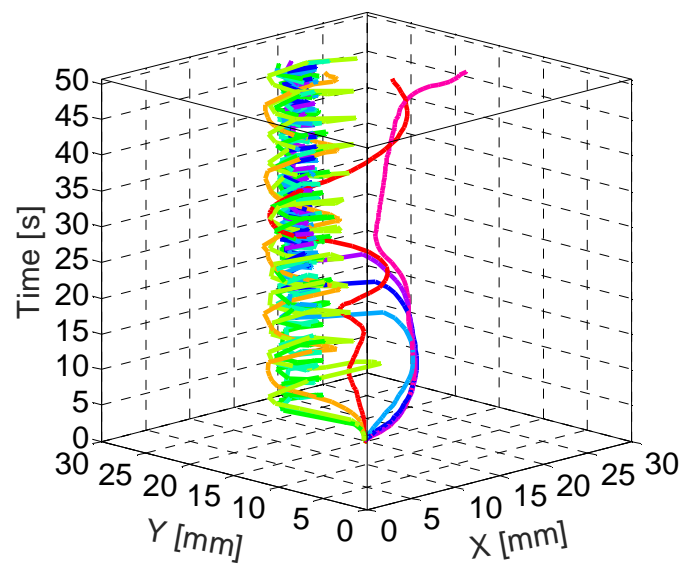

$-\mathrm{T}_{2}=20^{\circ} \mathrm{C}$

$-\mathrm{T}_{2}=22^{\circ} \mathrm{C}$

$-\mathrm{T}_{2}=24^{\circ} \mathrm{C}$

$-\mathrm{T}_{2}=26^{\circ} \mathrm{C}$

$-\mathrm{T}_{2}=28^{\circ} \mathrm{C}$

$-\mathrm{T}_{2}=30^{\circ} \mathrm{C}$

$-\mathrm{T}_{2}=32^{\circ} \mathrm{C}$

$-\mathrm{T}_{2}=34^{\circ} \mathrm{C}$

$-\mathrm{T}_{2}=36^{\circ} \mathrm{C}$
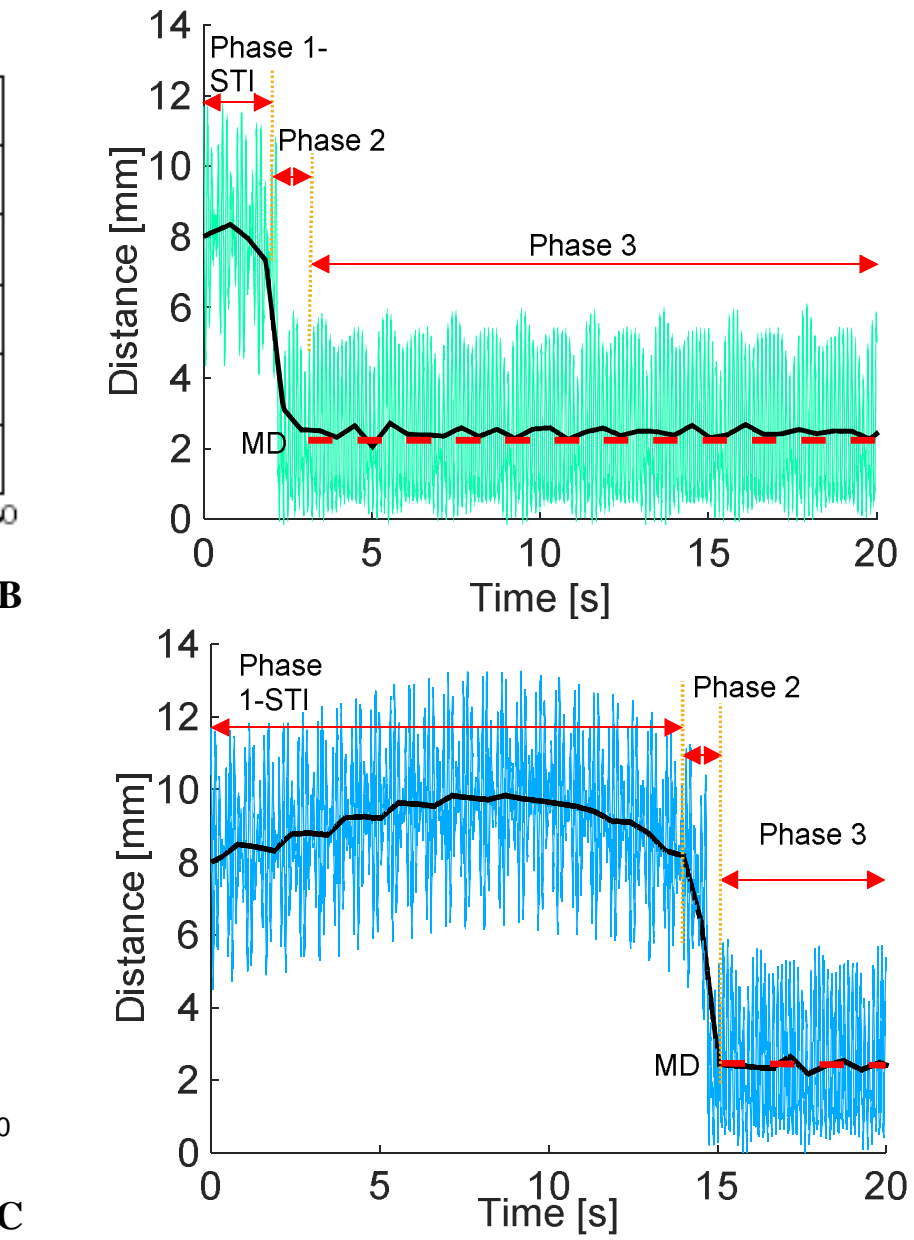

Fig. 6. Simulations of local perturbations of varying temperatures. A. Left - mean tip trajectories of a spiral wave in the presence of the local perturbations. Right - time-space plots of the trajectories. B-C. Detailed tip trajectories and mean trajectories (in black) for the cases of $\mathrm{T} 2=28^{\circ} \mathrm{C}$ and $\mathrm{T} 2=26^{\circ} \mathrm{C}$ ( $\mathrm{B}$ and $\mathrm{C}$, respectively). Left - the distance of the mean tip trajectory from the center of perturbation as a function of time. These traces show the typical 3 phases of drifting - slow transient phase (Phase 1), fast transient phase (Phase 2) and steady state phase (Phase 3). The measures of slow transient interval (STI) and mean distance at steady state (MD) are marked. 


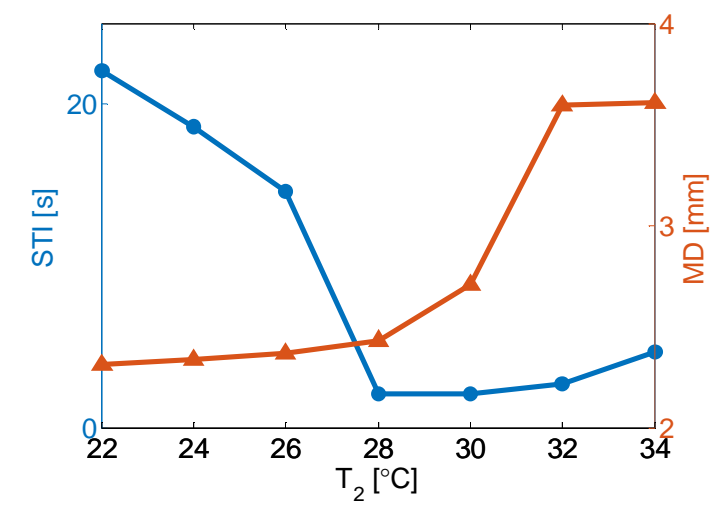

A

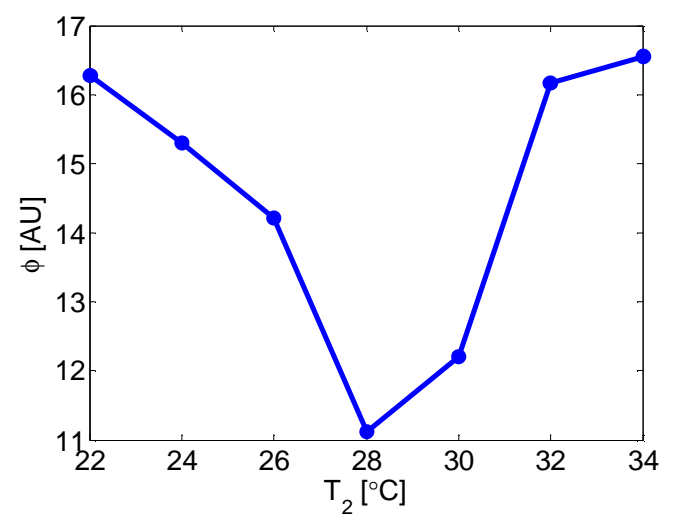

B

Fig. 7. Quality of spiral wave attraction as a function of perturbation temperature. A. Slow transient interval (STI) and mean distance (MD) of the spiral tip. B. Cost function, as defined in Eq. 7 , as a function of temperature, showing a clear optimal temperature of $28^{\circ} \mathrm{C}$.

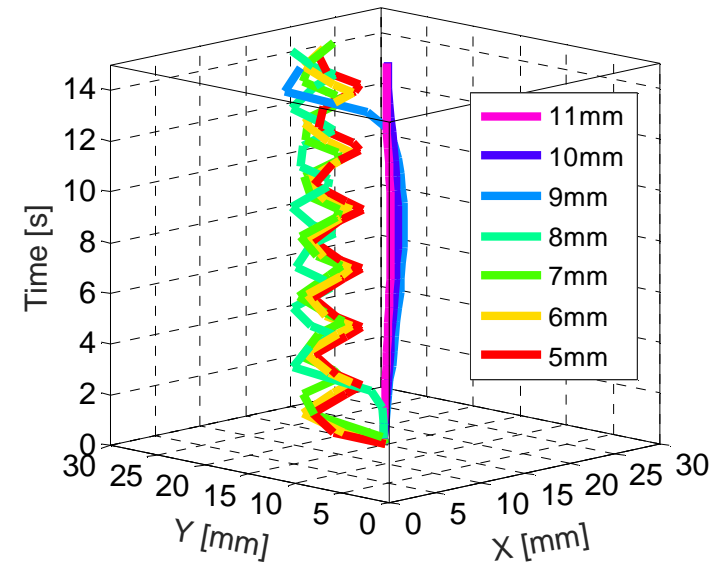

A

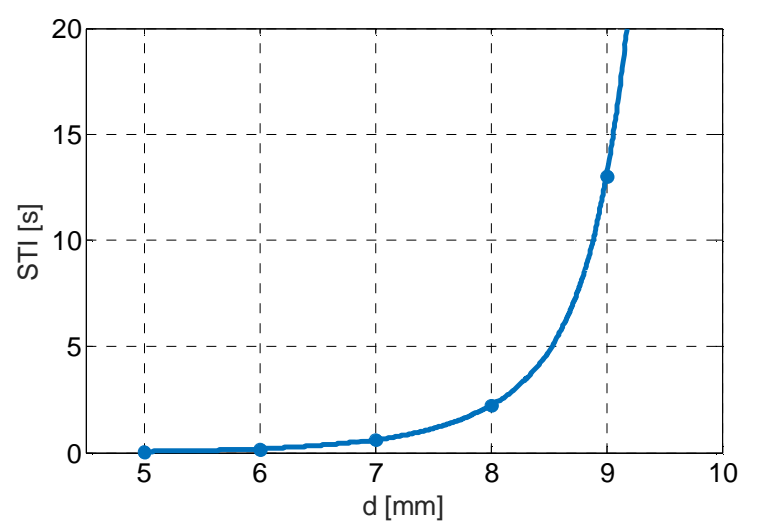

B

Fig. 8. Attraction of a spiral wave using a local perturbation of $T 2=28^{\circ} \mathrm{C}$ and varying locations. A. Time-space plots of the tip trajectory. B. Duration of the slow transient interval (STI) phase of attraction as a function of the initial distance, $d$, between the spiral wave core center and the center of perturbation. 


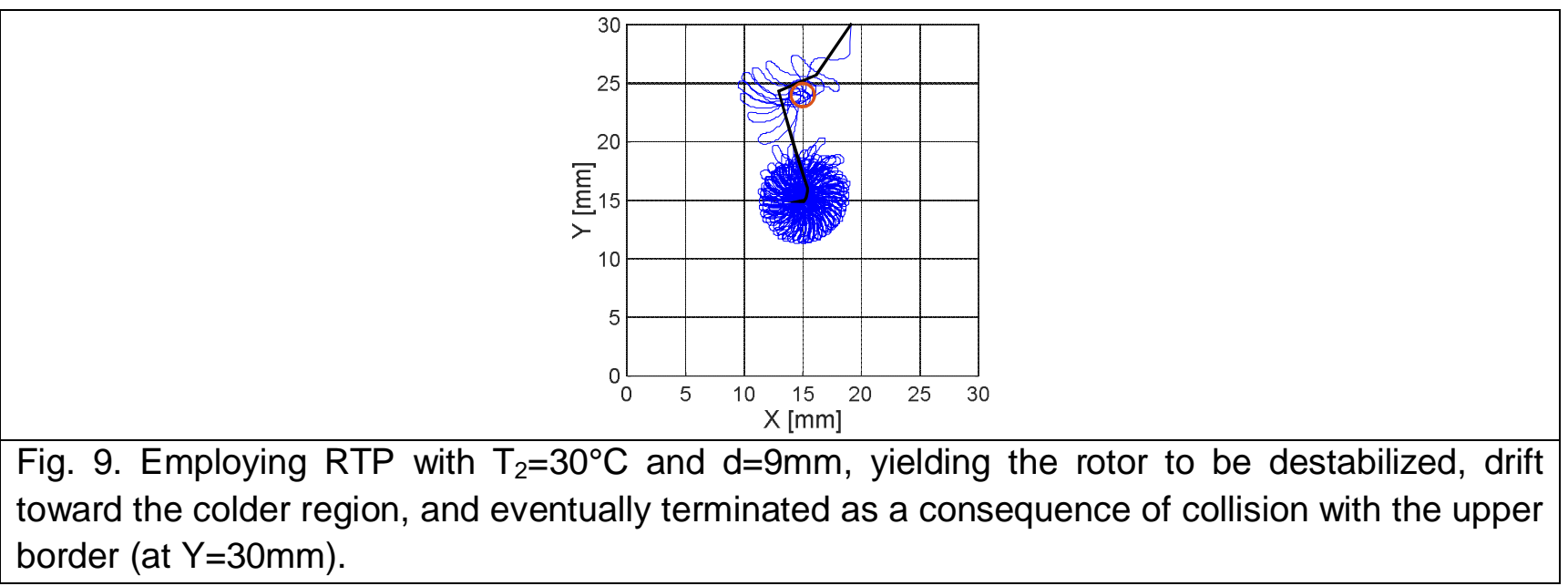

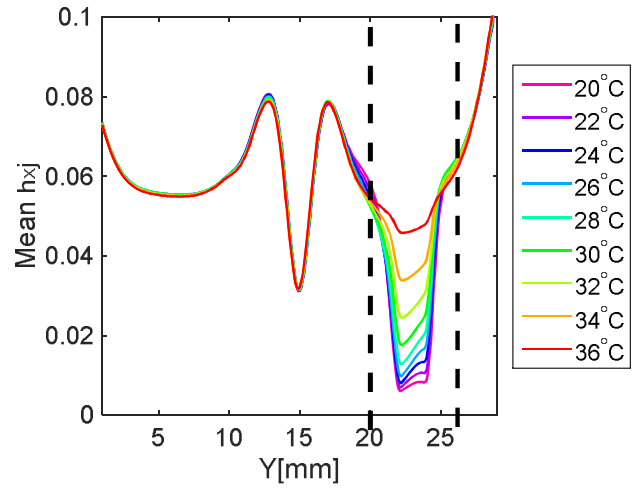

A

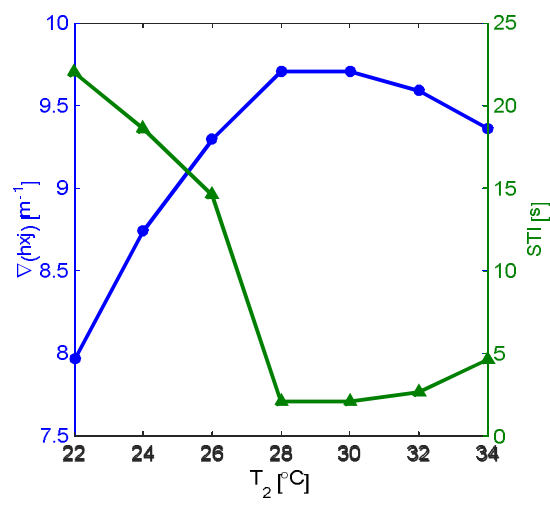

B

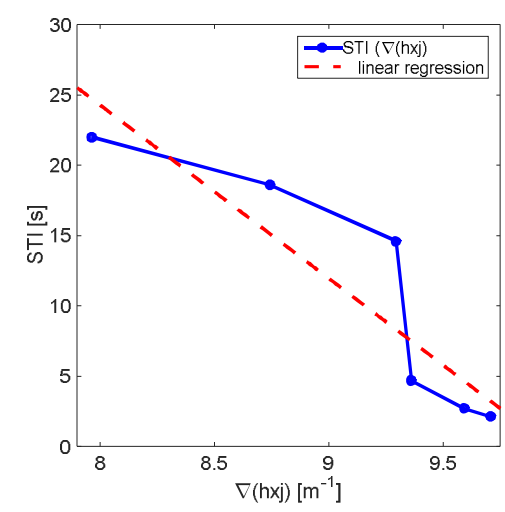

C

Fig. 10. Analysis of spiral wave drifting mechanism for a local temperature perturbation. A. Mean sodium channel availability along the dashed vertical line in Fig. 6B (left). The region between the two dashed black lines refers to the vertical distance between the spiral wave center and the perturbation center, and the gradient of excitability in that region was found to correlate with the drifting time of the spiral wave before anchoring to the perturbation. B. The gradient of excitability (between the two dashed lines in panel A) and the STI as a function of perturbation temperature. C. Relationship between the gradient of excitability and the STI reveals a decreasing monotonic function. 


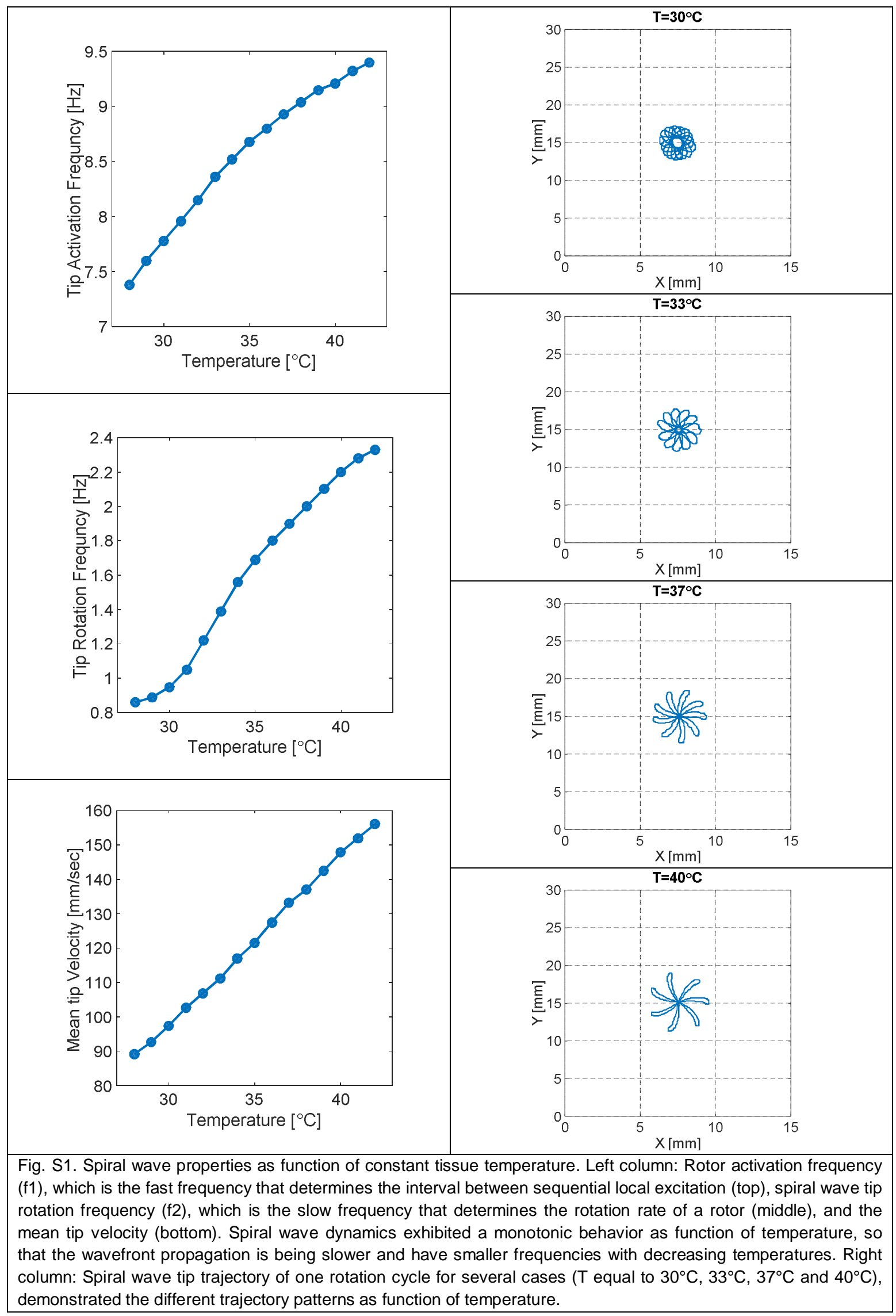



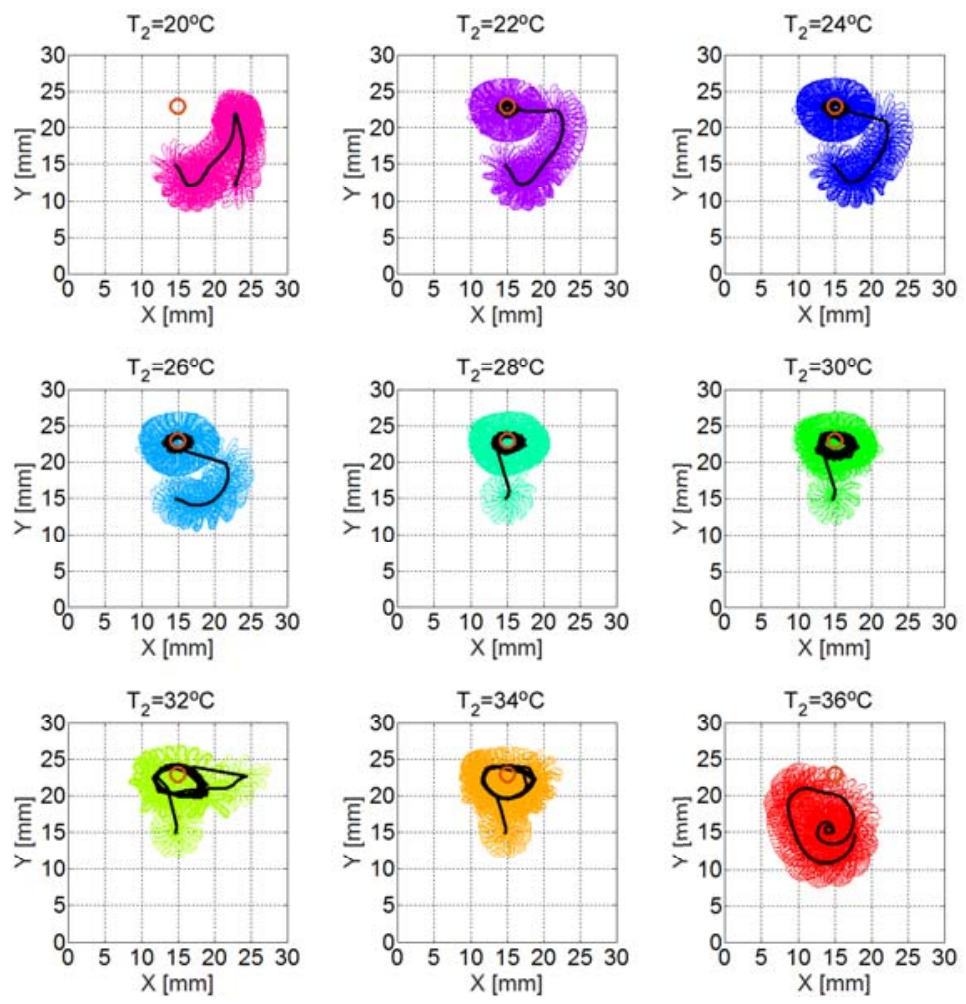

A
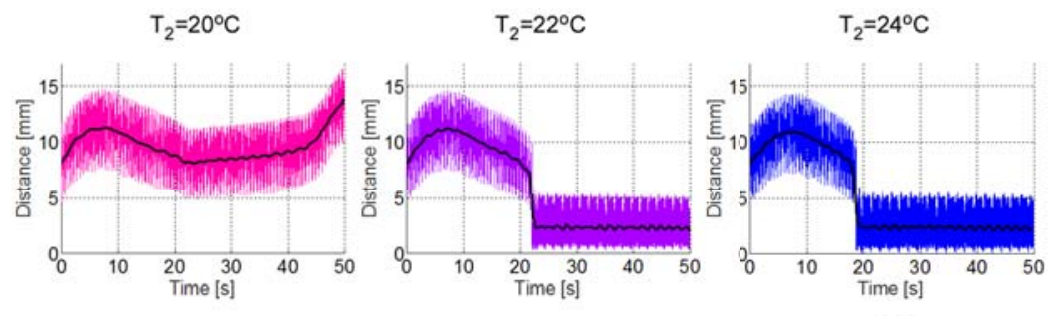

$\mathrm{T}_{2}=26^{\circ} \mathrm{C}$

$\mathrm{T}_{2}=28^{\circ} \mathrm{C}$

$\mathrm{T}_{2}=30^{\circ} \mathrm{C}$
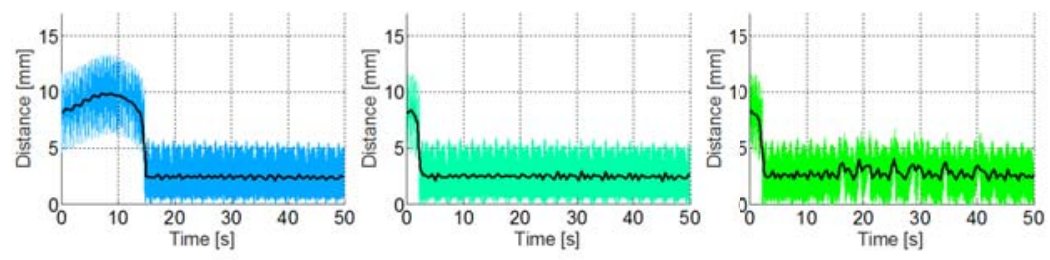

$\mathrm{T}_{2}=32^{\circ} \mathrm{C}$

$\mathrm{T}_{2}=34^{\circ} \mathrm{C}$

$\mathrm{T}_{2}=36^{\circ} \mathrm{C}$
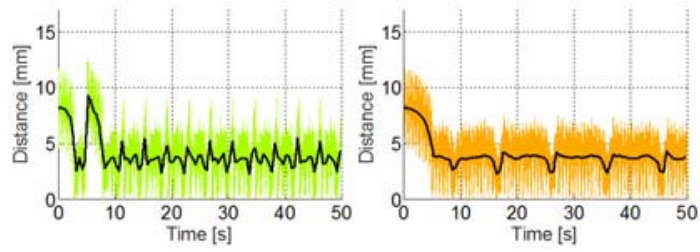

B

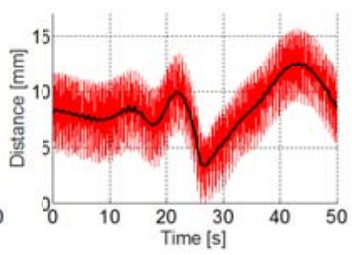

(3)

Fig. S2. Simulations of local perturbations of varying temperatures. A. Tip trajectories of a spiral wave in the presence of the local perturbations. In black - mean trajectories. Perturbation location is marked by a circle. B. Distance of the mean tip trajectory from the center of perturbation as a function of time. 

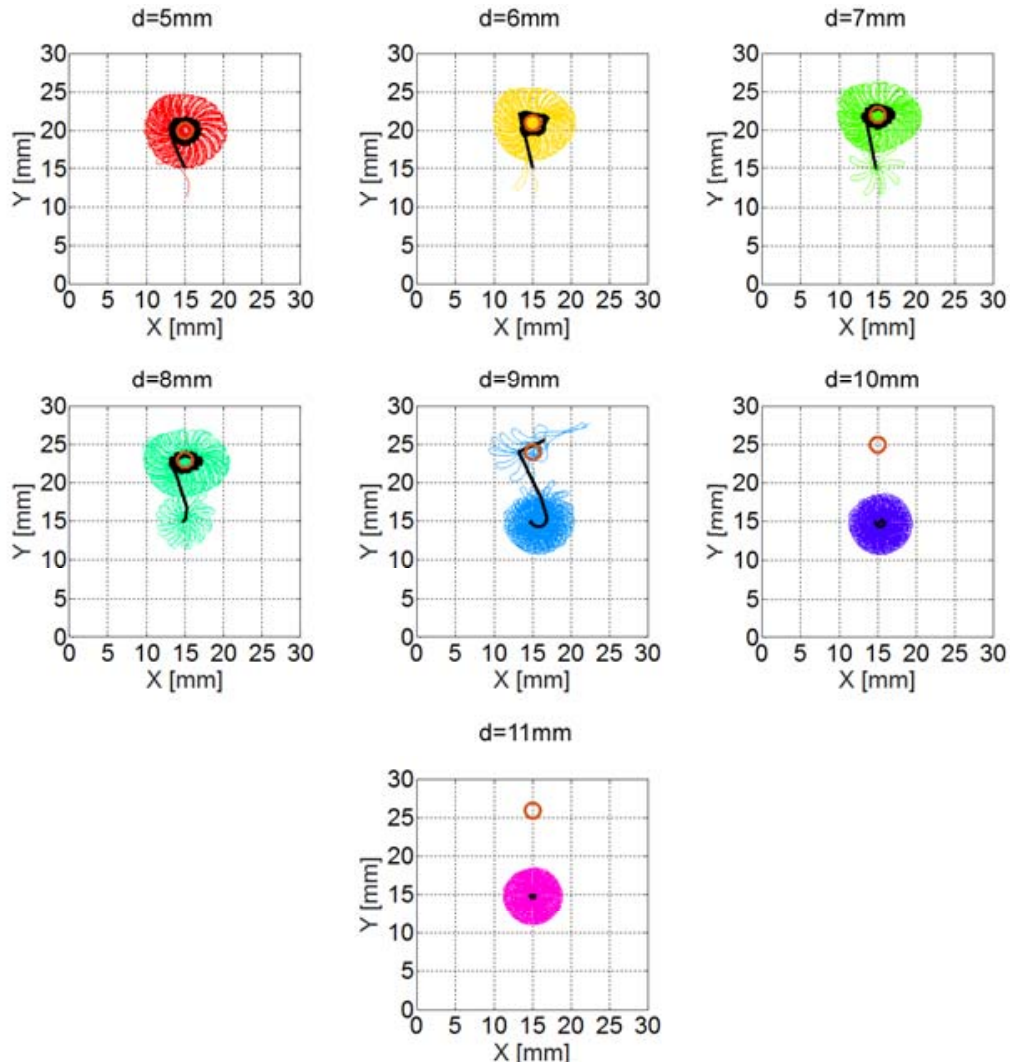

A
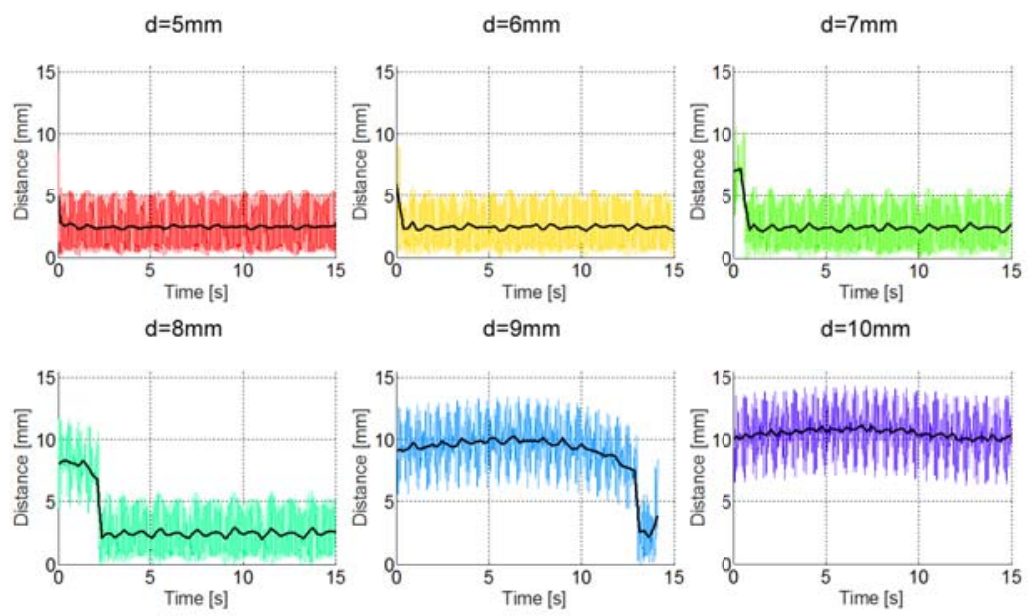

$\mathrm{d}=11 \mathrm{~mm}$

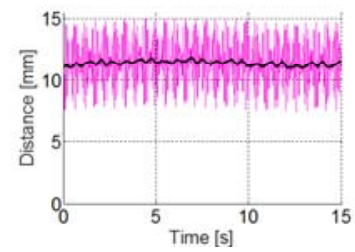

B

Fig S3. Simulations of local perturbations of varying initial distance from the spiral wave core. A. Tip trajectories of a spiral wave in the presence of the local perturbations. In black - mean trajectories. Perturbation location is marked by a circle. B. Distance of the mean tip trajectory from the center of perturbation as a function of time. 